\title{
Resistance loci affecting distinct stages of fungal pathogenesis: use of introgression lines for QTL mapping and characterization in the maize - Setosphaeria turcica pathosystem
}

\author{
Chia-Lin Chung ${ }^{1}$, Joy M Longfellow', Ellie K Walsh'1, Zura Kerdieh³, George Van Esbroeck', Peter Balint-Kurti5 and \\ Rebecca J Nelson*1,2
}

\begin{abstract}
Background: Studies on host-pathogen interactions in a range of pathosystems have revealed an array of mechanisms by which plants reduce the efficiency of pathogenesis. While R-gene mediated resistance confers highly effective defense responses against pathogen invasion, quantitative resistance is associated with intermediate levels of resistance that reduces disease progress. To test the hypothesis that specific loci affect distinct stages of fungal pathogenesis, a set of maize introgression lines was used for mapping and characterization of quantitative trait loci (QTL) conditioning resistance to Setosphaeria turcica, the causal agent of northern leaf blight (NLB). To better understand the nature of quantitative resistance, the identified QTL were further tested for three secondary hypotheses: (1) that disease QTL differ by host developmental stage; (2) that their performance changes across environments; and (3) that they condition broad-spectrum resistance.

Results: Among a set of 82 introgression lines, seven lines were confirmed as more resistant or susceptible than B73. Two NLB QTL were validated in $\mathrm{BC}_{4} \mathrm{~F}_{2}$ segregating populations and advanced introgression lines. These loci, designated qNLB1.02 and qNLB1.06, were investigated in detail by comparing the introgression lines with B73 for a series of macroscopic and microscopic disease components targeting different stages of NLB development. Repeated greenhouse and field trials revealed that $9 N L B 1.06_{T \times 303}$ (the Tx303 allele at bin 1.06) reduces the efficiency of fungal penetration, while $9 N L B 1.02_{B 73}$ (the B73 allele at bin 1.02) enhances the accumulation of callose and phenolics surrounding infection sites, reduces hyphal growth into the vascular bundle and impairs the subsequent necrotrophic colonization in the leaves. The QTL were equally effective in both juvenile and adult plants; qNLB1.06 $6_{\times 303}$ showed greater effectiveness in the field than in the greenhouse. In addition to NLB resistance, qNLB1.02 ${ }_{B 73}$ was associated with resistance to Stewart's wilt and common rust, while qNLB1.06 ${ }_{\text {T×303 }}$ conferred resistance to Stewart's wilt. The nonspecific resistance may be attributed to pleiotropy or linkage.

Conclusions: Our research has led to successful identification of two reliably-expressed QTL that can potentially be utilized to protect maize from S. turcica in different environments. This approach to identifying and dissecting quantitative resistance in plants will facilitate the application of quantitative resistance in crop protection.
\end{abstract}

\section{Background}

Pathogenesis is the series of events that occurs in a hostpathogen interaction, including infection and coloniza-

* Correspondence: rjn7@cornell.edu

1 Dept. of Plant Pathology and Plant-Microbe Biology, Cornell University, Ithaca, NY 14853, USA

Full list of author information is available at the end of the article tion of the host, and reproduction and dissemination of the pathogen. Genetic variation in host and/or pathogen can have quantitative or qualitative effects on the extent of disease. Many plant genetic factors that modulate pathogenesis have been discovered. The best known group is the R-genes, which provide high levels of resistance or even complete immunity. R-gene mediated resis- 
tance is initiated through a gene-for-gene interaction; the recognition of a pathogen effector by a host protein encoded by the R-gene leads to the induction of the hypersensitive response (HR), the production of antimicrobial metabolites such as phytoalexins, and the expression of pathogenesis-related (PR) proteins [1]. This type of interaction, typically resulting in a highly effective but race-specific defense response against pathogenic invasion, is sometimes known as qualitative resistance. Quantitative resistance, on the other hand, confers intermediate levels of resistance and is believed to be controlled by a set of genes distinct from, or partially overlapping with, those involved in qualitative resistance [2-7].

Although each quantitative resistance locus conditions a relatively small effect on pathogenesis, this type of resistance is of agricultural interest because qualitative resistance tends to be ephemeral in many pathosystems and is unavailable in others. Quantitative resistance is presumably more durable because multiple genes with minor effects lead to lower selection pressure and greater complexity to overcome [8]. A large number of quantitative trait loci (QTL) for disease resistance have been mapped in plants [6,9], but little is known about the underlying genetic basis or defense mechanisms involved. A range of genetic mechanisms controlling basal resistance, defense signalling pathways, detoxification, morphology, and development in the plant host, is hypothesized to be associated with reducing disease progress [6]. A small number of quantitative resistance genes have recently been cloned $[3-5,7,10]$, implicating diverse host functions in quantitative resistance.

Given that diverse host functions affect quantitative resistance, it is likely that QTL act at different stages of pathogenesis. The ways in which quantitative resistance affects different stages of pathogenesis has been addressed, to a limited extent, by comparing trait values obtained using distinct (usually macroscopic) disease components. In most (or probably all) of the phytopathosystems analyzed to date, differences in various disease parameters can be observed among plant genotypes. Previous QTL studies for foliar diseases have mapped distinct loci associated with incubation period, lesion number, lesion size, or diseased leaf area, with results suggesting that defense genes affecting lesion formation and lesion expansion may not be the same. In breeding programs, selection for decreased lesion length or lesion numbers can have insignificant effects on incubation period or disease severity (eg. [11]). These observations suggest that distinct resistance mechanisms govern different macroscopic components of resistance.

More insights into the role of a given disease QTL in limiting pathogenesis can be gained through histopathological analysis. While biochemical and microscopic anal- yses have been applied to investigate major gene resistance and fungal pathogenicity factors (reviewed by Vidhyasekaran [12]), few studies have reported the effect of individual QTL on distinct stages of pathogenesis from a microscopic view (exceptions include [5,13]). If QTL effective at specific stages of pathogenesis can be identified, combining favorable alleles for complementary QTL (eg. for infection and colonization) will likely provide greater levels of resistance.

Northern leaf blight (NLB; also known as turcicum blight and northern corn leaf blight) of maize was used as a model system to identify and characterize disease QTL at the macroscopic and microscopic levels. NLB, caused by Setosphaeria turcica (anamorph Exserohilum turcicum, syn. Helminthosporium turcicum), is one of the most prevalent foliar diseases in most maize-growing regions of the world. The disease causes periodic epidemics associated with significant yield losses [14-17], particularly under conditions of moderate temperature and high humidity [18]. Qualitative and quantitative forms of resistance against $S$. turcica are available in maize germplasm $[19,20]$, and have been widely utilized alone or in combination in resistance breeding programs [21]. A few histological studies have revealed the pathogenesis of $S$. turcica on maize leaves by staining, whole mount and serial dissection [22-25]. Marked phenotypic variation in symptom development has been observed among diverse maize lines in our multiple field and greenhouse trials. How macroscopic and microscopic phenotypes relate to specific QTL remains to be determined.

To answer questions concerning individual QTL effects, such as testing the hypothesis that distinct QTL act at different stages of pathogenesis, well-defined genetic stocks that differ only at specific loci are required. Introgression lines have been successfully used to study QTL in maize [26,27], rice [28], barley [29,30], tomato [31], and Arabidopsis [32]. While QTL analysis using recombinant inbred lines (RILs) provides greater statistical power in detecting QTL [33], RIL-based approaches have limitations in estimating QTL effects [31,32,34]. Introgression lines can be efficiently used to produce near-isogenic lines (NILs), which permit careful analysis of phenotypic effects associated with introgressed segments $[27,31]$.

NILs allow many long-standing questions about quantitative disease resistance to be addressed, such as the relationship between disease QTL and plant maturity, the interaction of QTL and environmental factors, and the specificity of resistance conditioned by QTL. The interplay between disease resistance and plant development has been widely recognized [35] yet remains poorly understood. In general, the resistance in adult plants or older leaves is greater than in juvenile plants or younger leaves (eg. [36-38]), and a correlation between resistance 
and flowering time has been found [[39], R. Wisser, J. Kolkman, and P. Balint-Kurti, unpublished]. Some QTL effects may thus be specific to certain plant developmental stages. In addition, the expression and effectiveness of many genes/QTL have been observed to be regulated by environmental conditions [9,40,41]. Another issue of fundamental and practical interest is whether a disease QTL confers specific or broad-spectrum resistance. A single locus can condition resistance to more than one disease, if it encompasses linked QTL effective against different diseases, or its underlying genes are involved in broadspectrum resistance pathways.

Here, we describe the use of introgression lines and derived NILs for QTL mapping and macro-/microscopic characterization in the maize - S. turcica pathosystem. We used an available population of introgression lines named TBBC3 [27]. This population is composed of introgression lines, each of which carries one or a few chromosomal segments of the donor genotype Tx303 in the genetic background of the recurrent parent B73. To better understand the nature of quantitative resistance, we assembled a panel of conventional and novel disease components targeting different stages of disease development. These were used to demonstrate that two QTL affect different stages of pathogenesis. The QTL were further characterized to shed light on three secondary hypotheses: (1) that disease QTL differ by host developmental stage (young versus adult plants); (2) that their performance changes across environments (field versus greenhouse); and (3) that they condition broad-spectrum resistance. This approach to identifying and dissecting quantitative resistance in plants will facilitate more effective and efficient application of quantitative resistance in crop protection.

\section{Methods \\ Plant materials}

A set of 82 TBBC3 introgression lines was provided by J. Holland of the USDA-ARS unit at North Carolina State University. The TBBC3 (for Tx303 by $\underline{B} 73$ Backcross $\underline{3}$ ) population, originally developed by C. Stuber at North Carolina State University [26], was the most extensively developed set of introgression lines available at the time for public use in maize. The population was derived from an initial cross of Tx303 and B73, followed by backcrossing to B73 for three generations. Each line was then selfed for several generations to attain homozygosity. Genotypic information was publicly available for each line, consisting of 14 restriction fragment length polymorphism (RFLP) and 116 simple sequence repeat (SSR) markers across the genome. Each line was known to carry one or more Tx303 introgressions, covering on average $2.5 \%$ of the genome, in the background of the sequenced refer- ence maize line B73. Taken together, the set of introgression lines collectively carries $\sim 89 \%$ of Tx303 genome [27].

To validate and characterize the effects of Tx303 introgressions, several $\mathrm{BC}_{4} \mathrm{~F}_{2}$ populations were developed by crossing selected TBBC3 lines to $\mathrm{B} 73$. Sets of $\mathrm{BC}_{4} \mathrm{~F}_{3}$ and $\mathrm{BC}_{4} \mathrm{~F}_{4}$ lines carrying different introgression(s) were subsequently derived by single-seed descent. After four generations of marker-assisted backcrossing, the $\mathrm{BC}_{4} \mathrm{~F}_{3}$ and $\mathrm{BC}_{4} \mathrm{~F}_{4}$ lines were designated as NILs.

\section{Assessments of northern leaf blight}

A single isolate of $S$. turcica (NY001, race 1) was used in the experiments carried out in New York (NY), and a mixture of isolates representing race 1 , race 23 , and race $23 \mathrm{~N}$ of $S$. turcica was used in North Carolina (NC). For preparation of liquid inoculum, S. turcica was cultured for two to three weeks on lactose - casein hydrolysate agar (LCA) plates under a $12 \mathrm{hr} / 12 \mathrm{hr}$ normal light-dark cycle at room temperature. The conidia were then dislodged with sterile $\mathrm{ddH}_{2} \mathrm{O}$ and a glass rod, filtered through four layers of cheesecloth, and adjusted to the final concentration with the aid of a haemocytometer. Solid inoculum was prepared by culturing S. turcica on sorghum grains in plastic milk jugs for two to three weeks under the same condition. For each jug, $900 \mathrm{ml}$ of sorghum grains were soaked overnight in $600 \mathrm{ml}$ of water in a milk jug. The jug was then autoclaved twice at $121^{\circ} \mathrm{C}, 15$ $\mathrm{lb} / \mathrm{cm}^{2}$, for 25 minutes per run. The jugs were inoculated by dividing the spore suspension produced from one heavily colonized LCA plate $(10 \mathrm{~cm}$ diameter) among five jugs. Jugs were shaken daily to prevent caking and accelerate fungal colonization.

In this study, the "juvenile" phase refers to earlier vegetative stages, and the "adult" phase refers to later vegetative stages and reproductive stages. Juvenile plants (at the five- to six-leaf stage) and adult plants (late vegetative stage, around two weeks before tasselling) were used for inoculation. The inoculation technique utilized depended on the specific objectives of the experiment. In the field trials in NY, plants were inoculated with both liquid $(0.5$ $\mathrm{ml}$ of spore suspension, $4 \times 10^{3}$ conidia per $\mathrm{ml}, 0.02 \%$ Tween 20$)$ and solid inoculum (1/4 teaspoon, $\sim 1.25 \mathrm{ml}$ of colonized sorghum grains) placed in the whorl. This was done to ensure the viability of inoculum across a range of conditions (under optimal conditions, the liquid inoculum was considered most effective, while the solid inoculum was considered to perform more effectively under dry conditions). In the field trials in NC, $\sim 20$ grains of sorghum colonized with $S$. turcica were placed in the whorl.

In greenhouse trials, whorl inoculation with aforementioned liquid inoculum was carried out for assessing individual plants in the segregating populations. Spray 
inoculation was performed for detailed QTL characterization using NILs, as it provides significantly better differentiation for NLB evaluation (data not shown). The spray method was preferred for microscopic examination and real-time PCR quantification. A higher number of spores could be evenly distributed on leaf surface with spraying, making the subsequent sampling more effective and accurate. About $0.5 \mathrm{ml}$ of concentrated spore suspension $\left(5 \times 10^{4}\right.$ conidia per $\mathrm{ml}, 0.02 \%$ Tween 20$)$ was evenly sprayed on the first fully expanded leaf with an airbrush (Badger Model 150) at 20 psi. After inoculation, the plants were kept overnight in a mist chamber at $>85 \%$ $\mathrm{RH}$, then maintained at $22^{\circ} \mathrm{C}$ day $/ 18^{\circ} \mathrm{C}$ night temperature with a $14 \mathrm{hr}$-light/10 hr-dark cycle.

\section{Phenotypic characterization of resistance to NLB}

Field experiments were conducted at Cornell's Robert Musgrave Research Farm in Aurora, NY and Central Crops Field Station in Clayton, NC. Plants were evaluated for different disease parameters and for days to anthesis (DTA). DTA, which was only assessed for field-grown plants, was scored on a row basis when $>50 \%$ of the plants in a row started to shed pollen. An overview of various disease components used in this study and their corresponding stages during NLB development is summarized in Table 1. The evaluation method for each parameter is illustrated as below.

\section{Microscopic analysis}

Two microscopy techniques were applied to investigate differential development of S. turcica in the NILs: trypan blue staining and $\mathrm{KOH}$-aniline blue staining. In greenhouse trials, infected leaf samples were harvested from individual plants. In the field trial, for the purpose of obtaining a sufficient number of infection sites for examination, samples (per genotype per block) were collected from four plants in a row and pooled for subsequent treatments.

Trypan blue staining was performed as previously described $[25,42]$ with some modifications. Infected tissues were collected at two days post inoculation (dpi) from plants in the greenhouse, and at $3 \mathrm{dpi}$ from plants in the field trials. Leaf samples were cut into $1 \times 1 \mathrm{~cm}^{2} \mathrm{seg}$ ments, cleared first in an acetic acid: ethanol (1:3, v/v) solution overnight, then in an acetic acid: ethanol: glycerol $(1: 5: 1, \mathrm{v} / \mathrm{v} / \mathrm{v})$ solution for at least 3 hours. The samples were subsequently incubated overnight in a staining solution of $0.01 \%(\mathrm{w} / \mathrm{v})$ trypan blue in lactophenol, and rinsed then stored in $60 \%$ glycerol until examination. Specimens were transferred onto microscopic slides and examined under a compound microscope. Fifty to 60 germinated conidia were assessed per individual plant (greenhouse) or per row (field).

A modified $\mathrm{KOH}$-aniline blue fluorescence technique [43] was used to visualize the growth of fungal hyphae inside the infected leaves and the accumulation of (plantproduced) callose and phenotypic compounds around the infection sites. Infected leaves were sampled at $4 \mathrm{dpi}$ and $7 \mathrm{dpi}$ in greenhouse trials, and at $6 \mathrm{dpi}$ in the field trial. The samples were cut into $1 \times 1 \mathrm{~cm}^{2}$ segments, incubated in $1 \mathrm{M} \mathrm{KOH}$ at room temperature for 24 hours, then autoclaved at $121^{\circ} \mathrm{C}, 15 \mathrm{lb} / \mathrm{cm}^{2}$ for $2-5 \mathrm{~min}$. Autoclaving time was adjusted according to the rigidity of leaves, which varied with plant genotype and maturity. The autoclaved specimens were rinsed in $\mathrm{ddH}_{2} \mathrm{O}$ three times, then stored in autoclaved $\mathrm{dd}_{2} \mathrm{O}$ until examination. Specimens were carefully placed on microscopic slides and mounted in a staining solution of $0.05 \%$ aniline blue in $0.067 \mathrm{M} \mathrm{K}_{2} \mathrm{HPO}_{4}$ (prepared at least $2 \mathrm{hrs}$ prior to use). Thirty five to 40 germinated conidia were checked per individual plant (greenhouse) or per row (field) under a Zeiss fluorescence microscope with a G365 excitation filter, a FT395 dichromatic beam splitter, and an LP420 barrier filter.

\section{Quantitative real-time PCR for quantifying fungal colonization}

DNA-based quantitative PCR (qPCR) was performed as described by Qi and Yang [44] with some modifications. The specific pair of primers for S. turcica: forward: 5'TCTTTTGCGCACTTGTTGTT and reverse: 5'-CGATGCCAGAACCAAGAGAT, was designed based on the internal transcribed spacer 1 (ITS1) of ribosomal DNA gene in $S$. turcica. The ITS1 sequence (GenBank: AF163067.1) was obtained from the nucleotide database of the National Center for Biotechnology Information (NCBI). PCR amplification resulted in a specific fragment of 170 base pairs.

Inoculation experiments were performed three times in the greenhouse. Five plants per genotype were sprayinoculated. The same amounts of infected tissue $(0.12 \mathrm{~g}$ per plant) were collected at $9 \mathrm{dpi}$ from the middle part of each leaf. Leaf samples were ground with liquid nitrogen and DNA was extracted following the protocol described later. The extracted DNA from each individual plant was dissolved in $100 \mu \mathrm{l}$ TE buffer. Total DNA concentration was determined using the PicoGreen dsDNA quantitation assay kit (Invitrogen, Eugene, Oregon, USA). Fungal DNA was quantified using $\mathrm{qPCR}$. The ratio of fungal biomass in maize leaves was computed from the amount of fungal DNA divided by total DNA.

Each qPCR reaction was performed in a total volume of $25 \mu \mathrm{l}$, containing $12.5 \mu \mathrm{l}$ of iTaq SYBR ${ }^{\circ}$ Green Supermix with ROX (Bio-Rad Laboratories, Hercules, CA, USA), 3 $\mu \mathrm{l}$ of 7.5-fold diluted DNA from an infected plant and 300 $\mathrm{nM}$ of each forward and reverse primer. PCR samples were incubated in an ABI Prism 7000 Sequence Detection System (Applied Biosystems, Foster City, CA, USA) with thermal cycling parameters of $95^{\circ} \mathrm{C}$ for 2 min fol- 
Table 1: Overview of disease components used to target different stages of northern leaf blight (NLB) development.

\begin{tabular}{|c|c|c|c|c|}
\hline Disease component & Description (unit) & Targeted disease development stage(s) & Evaluation & Literature \\
\hline $\begin{array}{l}\text { Incidence of multiple } \\
\text { appressoria }^{a}\end{array}$ & $\begin{array}{l}\text { The incidence of }>1 \text { appressorium } \\
\text { developed from each germinated } \\
\text { conidium (\%) }\end{array}$ & Pre-penetration & $\begin{array}{l}\text { Trypan blue staining and } \\
\text { microscopy }\end{array}$ & {$[25,42]$} \\
\hline Infection efficiency & $\begin{array}{l}\text { The incidence of successful } \\
\text { infection per germinated } \\
\text { conidium (\%) }\end{array}$ & Penetration into the epidermal cell & $\begin{array}{l}\text { Trypan blue staining and } \\
\text { microscopy, } \mathrm{KOH} \text {-aniline blue } \\
\text { fluorescence microscopy }\end{array}$ & {$[25,42,94]$} \\
\hline $\begin{array}{l}\text { Accumulation of } \\
\text { callose and } \\
\text { phenolics }\end{array}$ & $\begin{array}{l}\text { Diameter of enhanced fluorescing } \\
\text { area surrounding the infection } \\
\text { site }(\mu \mathrm{m})\end{array}$ & $\begin{array}{l}\text { Intercellular and intracellular hyphal } \\
\text { growth from primary infected cell } \\
\text { to surrounding mesophyll cells }\end{array}$ & $\begin{array}{l}\mathrm{KOH} \text {-aniline blue fluorescence } \\
\text { microscopy }\end{array}$ & [43] \\
\hline $\begin{array}{l}\text { Vascular invasion } \\
\text { efficiencya }\end{array}$ & $\begin{array}{l}\text { The incidence of hyphae entering } \\
\text { vascular bundles per } \\
\text { infection site (\%) }\end{array}$ & Hyphal growth into the vasculature & $\begin{array}{l}\mathrm{KOH} \text {-aniline blue fluorescence } \\
\text { microscopy }\end{array}$ & [43] \\
\hline Fungal biomass ratioa & $\begin{array}{l}\text { The percentage of fungal DNA } \\
\text { divided by the total DNA in the } \\
\text { infected leaf tissues (\%) }\end{array}$ & $\begin{array}{l}\text { Overall fungal growth in leaves before } \\
\text { the appearance of necrotic lesions }\end{array}$ & $\begin{array}{l}\text { DNA-based real-time } \\
\text { quantitative PCR }\end{array}$ & [44] \\
\hline Incubation period (IP) & $\begin{array}{l}\text { The number of days from } \\
\text { inoculation to the appearance of } \\
\text { the first lesion on a plant (days) }\end{array}$ & $\begin{array}{l}\text { Xylem plugging due to extensive } \\
\text { hyphal growth in the vascular veins }\end{array}$ & Visual examination & {$[73,74,76-78,95]$} \\
\hline Lesion expansion (LE) & $\begin{array}{l}\text { The longitudinal expansion of a } \\
\text { lesion per day }(\mathrm{mm})\end{array}$ & $\begin{array}{l}\text { Destructive hyphal growth in primary } \\
\text { inoculated leaves }\end{array}$ & Digital caliper measurement & {$[78,96]$} \\
\hline $\begin{array}{l}\text { Diseased leaf } \\
\text { area (DLA) }\end{array}$ & $\begin{array}{l}\text { The percentage of infected leaf } \\
\text { area of the entire plant, } \\
\text { disregarding decayed bottom } \\
\text { leaves (\%) }\end{array}$ & $\begin{array}{l}\text { Destructive hyphal growth on the } \\
\text { leaves of a entire plant, caused by } \\
\text { primary and secondary inoculum }\end{array}$ & Visual examination & {$[73,76,77,94,97]$} \\
\hline Disease severity ${ }^{b}$ & $\begin{array}{l}\text { The severity of infected leaf area } \\
\text { of the entire plant (scale 1-10, 1: } \\
\text { little diseased area) }\end{array}$ & $\begin{array}{l}\text { Destructive hyphal growth on the } \\
\text { leaves of a entire plant, caused by } \\
\text { primary and secondary inoculum }\end{array}$ & Visual examination & [98] \\
\hline $\begin{array}{l}\text { Area under the } \\
\text { disease progress } \\
\text { curve (AUDPC) }\end{array}$ & $\begin{array}{l}\text { Total area under the graph of DLA } \\
\text { or other disease severity rating } \\
\text { (area unit) }\end{array}$ & $\begin{array}{l}\text { An overall destructive hyphal growth } \\
\text { on a plant throughout the season }\end{array}$ & $\begin{array}{l}\text { Calculated from visual } \\
\text { examination scores }\end{array}$ & {$[76,77]$} \\
\hline
\end{tabular}

\footnotetext{
a To authors' knowledge, not previously utilized as a disease component for evaluating NLB resistance.
}

b Only applied in the 2006 trial in North Carolina. 
lowed by 40 cycles of $15 \mathrm{sec}$ at $95^{\circ} \mathrm{C}$ and $30 \mathrm{sec}$ at $56^{\circ} \mathrm{C}$. Two standard curves were constructed by mixing a series of $S$. turcica DNA $(0,1,10,50,100,500$ and $1000 \mathrm{pg})$ with $50 \mathrm{ng}$ of maize DNA extracted from non-inoculated B73 and Tx303 plants, respectively. The quantification of fungal biomass in infected B73 and derived NILs was based on the first standard curve (created from mixing with a constant amount of B73 DNA), while the quantification for DNA from infected Tx303 was based on the second standard curve. Three technical replicates were carried out in individual plates for both Picogreen quantification and iTaq SYBR Green PCR, with the samples for standard curves repeated twice in the same plates.

\section{Incubation period (IP)}

Individual plants were checked every day after $7 \mathrm{dpi}$ for the appearance of the first wilted lesions. The number of dpi when the first lesions were observed was scored as the IP. In the trials at Aurora NY, IP scores were rated for individual plants, then averaged for the rows. In the trial at Clayton NC, IP was recorded on a row basis when > $50 \%$ of the plants in a row started showing lesions.

\section{Lesion expansion (LE)}

LE was scored in the trials in NY but not NC. Around two to three weeks after inoculation, three lesions per plant were randomly chosen for measurement. Lesion margins were marked and then measured 10-14 days later for the longitudinal extension with a digital caliper. The expansion measurements taken from three lesions were averaged, and divided by the number of days from the marking until measurement of the lesions.

\section{Primary diseased leaf area (PrimDLA)}

Primary DLA was rated as the percentage of infected leaf area of the inoculated leaves in the 2006 trial in NY. It was scored once on a row basis at around three to four weeks after inoculation.

\section{Diseased leaf area (DLA)}

DLA was rated as the percentage of infected leaf area of the entire plant, disregarding decayed bottom leaves. DLA was rated on a row basis for TBBC3 lines and derived NILs, and on individual plants for testing traitmarker association in segregating populations. DLA was rated three to four times per season at 10-14 day intervals. The first DLA score was recorded at one to two weeks after observing the onset of secondary infection.

\section{Disease severity}

Disease severity was rated on a row basis four times through the season in the 2006 trial in NC. The severity score was based on a 1 to 9 scale corresponding to the percentage of infected leaf area on primarily the ear leaf as well as the leaves above and below the ear leaf (severity 1: $0 \%, 2: 12.5 \%, 3: 25 \%, \ldots . ., 9: 100 \%)$.

\section{Area under the disease progress curve (AUDPC)}

The AUDPC was calculated as $\sum_{i=1}^{n-1} \frac{\left(y_{i}+y_{i+1}\right)\left(t_{i+1}-t_{i}\right)}{2}$, where $y_{i}=$ DLA or disease severity at time $i, t_{i+1}-t_{i}=$ day interval between two ratings, $n=$ number of ratings [45].

\section{Evaluation for multiple disease resistance Stewart's wilt}

Pantoea stewartii (syn. Erwinia stewartii) strain PsNY003, originally collected in NY in 1991, was obtained from Helene Dillard of Cornell University. Inoculum was prepared as previously described [46] with the use of nutrient agar plates and nutrient broth as media. Plants at the five- to six-leaf stage were inoculated with $P$. stewartii following the pinprick method $[47,48]$ with a modified inoculator. The multiple-pin inoculator was made with 30 T-pins (1.5 inch long), pieces of $5.5 \mathrm{~cm} \times$ $6.5 \mathrm{~cm}$ sponge and cork board (3/8 inch thick) fastened on two arms of a tong with rubber bands. Primary diseased leaf area was rated as the percentage of infected area of the inoculated leaves. It was scored twice (two and three weeks after inoculation) on a row basis and the scores were averaged.

\section{Anthracnose stalk rot}

A New York isolate of Colletotrichum graminicola (teleomorph: Glomerella graminicola) (isolate Cg151) was obtained from Gary Bergstrom of Cornell University. Each plant was inoculated with $1 \mathrm{ml}$ of $10^{6}$ conidia per ml $(0.02 \%$ Tween 20$)$ when more than $50 \%$ of the plants in every row were tasseling [49]. Inoculum preparation and inoculation were conducted as described by MuimbaKankolongo and Bergstrom [50], with the replacement of plastic straw with $1 \mathrm{ml}$ pipette tips. Four weeks after inoculation, stalks were split longitudinally and the percentages of discolored area of individual internodes were visually rated [49] and summed for analysis. In 2007, eight consecutive internodes were scored from four plants per row (inoculation was conducted in NLB plot); in 2008, six consecutive internodes were scored from eight plants per row.

\section{Common smut}

In the 2007 trial, plants in NLB plots were evaluated for the development of ear galls and stalk galls resulting from natural infection. Artificial inoculation was conducted in the 2008 trial using six compatible strains of Ustilago maydis (UmNY001, UmNY002, UmNY003, UmNY004, UmNY008 and UmNY009), which were isolated from naturally infected smut galls collected at Aurora NY in 2007. U. maydis strains were cultured as described by du Toit and Pataky [51]. Inoculum was prepared by adjusting sporidial suspension (in potato dextrose broth) to a final 
concentration of $10^{6}$ sporidia per $\mathrm{ml}(0.02 \%$ Tween 20$)$ with sterilized distilled water. Equal amounts of sporidia from the six compatible strains were mixed prior to inoculation. Non-pollinated (shoot-bagged) primary ear of each plant was injected with $2 \mathrm{ml}$ of mixed sporidial suspension at the time that the silks of most primary ears had emerged $1-5 \mathrm{~cm}$. Every plant in the row was rated from four weeks after inoculation for ear galls on a $0-10$ scale, corresponding to the number and size of galls and the disease severity of the entire plant $(0=$ no smut galls and $10=$ a dead smut-infected plant). Stalk galls resulting from natural infection were also scored.

\section{Common rust}

Urediniospores of Puccinia sorghi were collected from naturally infected leaves at Aurora NY in 2007. Inoculum was increased on three- to four-leaf stage seedlings of susceptible sweet corn in the greenhouse. About $300 \mathrm{mg}$ of stock urediniospores (preserved at $-80^{\circ} \mathrm{C}$ ) were suspended in $100 \mathrm{ml}$ of Sortrol oil (Chevron Phillips Chemical Company, Phillips, TX, USA) [52] and evenly applied on leaves with a spray gun (Preval, Yonkers, NY, USA). Plants were kept overnight in a mist chamber at $>85 \%$ $\mathrm{RH}$, then grown for two more weeks until Puccinia sorghi sporulated vigorously. The urediniospores were collected by agitating infected leaves with mature rust pustules in distilled water and filtering through four layers of cheesecloth. Spore suspension was adjusted to a final concentration of $2 \times 10^{5}$ urediniospores per $\mathrm{ml}$ with the aid of a haemocytometer. Field plants were inoculated at the sixto eight-leaf stage by adding $1 \mathrm{ml}$ of spore suspension (0.02\% Tween 20) in the whorl [53]. Evaluation for disease severity was based on a 0-10 scale with 0.5 increments, corresponding to the percentage of infected leaf area of the entire plant $(0=$ no disease, $1=10 \%, \ldots, 10=100 \%)$. Disease severity was scored three times at 9-day intervals from four weeks after inoculation in 2008, and at 15-20 day intervals from 10 days after inoculation in 2009. AUDPC was calculated from the three severity scores as described above.

\section{DNA extraction and genotyping}

About $0.1 \mathrm{~g}$ of fresh or lyophilized leaf tissue and a stainless steel ball (5/32 inch diameter, OPS Diagnostics, NJ, USA) were loaded in each well of a 96-well plate (Corning Costar 96 Well Polypropylene Cluster Tubes). Tissues were frozen at $-80^{\circ} \mathrm{C}$ and pulverized at 450 strokes $/ \mathrm{min}$ for 50-120 sec using Genogrinder 2000 (SPEX CertiPrep Inc., Metuchen, NJ, USA). Genomic DNA was extracted following a standard $\mathrm{CTAB}$ extraction protocol $[54,55]$ with $500 \mu \mathrm{l}$ of CTAB extraction buffer, $400 \mu \mathrm{l}$ of chlorophorm/isoamyl alcohol $(24: 1, \mathrm{v} / \mathrm{v})$, and $300 \mu \mathrm{l}$ of isopropanol. Fungal DNA was extracted as described, except that S. turcica mycelium was ground in liquid nitrogen with a pestle and mortar, and transferred to a $1.5 \mathrm{ml}$ eppendorf tube for later steps.

Simple sequence repeat (SSR) markers were used for genotypic analysis following a single-reaction nested PCR method [56]. Each PCR reaction was performed as described by Wisser et al. [57] in a total volume of $13 \mu \mathrm{l}$, with the same thermal cycling parameters as described by Schuelke [56]. Amplicons labeled with different fluorescent dyes were multiplexed (combining up to four PCR reactions, $0.7 \mu \mathrm{l}$ PCR product per specific primer pair), mixed with $9 \mu \mathrm{l}$ formamide and 0.05-0.1 $\mu \mathrm{l} \mathrm{GeneScan-500}$ LIZ size standard (Applied Biosystems), and analyzed on the Applied BioSystems 3730xl DNA Analyzer at Biotechnology Resource Center at Cornell University. The sizes of amplicons were scored using GeneMapper v. 3.0 (Applied Biosystems).

\section{Experimental design}

The full set of 82 TBBC3 lines, a subset of 15 TBBC3 lines, and derived sets of $\mathrm{BC}_{4} \mathrm{~F}_{3}$ and $\mathrm{BC}_{4} \mathrm{~F}_{4}$ NILs were evaluated in the field following the resolvable incomplete block design (also known as an alpha design [58]). The 82 TBBC3 lines were evaluated at Aurora NY for IP, PrimDLA, DLA, and DTA. To precisely estimate the effects of Tx303 introgressions, every experimental row was grown next to a row of B73 (B73 in every third row). The whole design had two replicates with 14 blocks per replicate, and six experimental rows plus three B73 rows per block. A parallel experiment was conducted on the 82 TBBC3 lines at Clayton NC with two replicates, nine blocks per replicate and 10 rows per block. The $B 73$ rows were included in every other block for the control (five B73 rows per replicate). Disease severity and DTA were evaluated. The field trials on the 15 selected TBBC3 lines were conducted at Aurora NY and Clayton NC following the alpha design with B73 in every third row as described above. The 15 lines were selected from those significantly different from B73 in NLB resistance (based on the firstyear results), and/or from the lines carrying Tx303 introgression(s) corresponding to previously identified NLB QTL. In each location, there were four replicates with four blocks per replicate, and four experimental rows plus two B73 rows per block. The IP, DLA, and DTA were evaluated. An additional disease component, lesion expansion (LE), was evaluated at Aurora NY as well. During the same field season, to understand the effectiveness of identified QTL in adult plants, the same 15 selected TBBC3 lines were grown in a separate field plot at Aurora NY, following identical arrangement and design with three replicates. The plants were inoculated two weeks before flowering (rather than the usual five- to six-leaf stage), and evaluated for IP and DLA.

To associate resistance with specific introgressions, several $\mathrm{BC}_{4} \mathrm{~F}_{2}$ populations were genotyped and pheno- 
typed for IP, LE, and DLA in either greenhouse or in the field at Aurora NY. Individual plants of each population were grown within a single block with $\mathrm{B} 73$ rows as the border. For QTL confirmation, derived $\mathrm{BC}_{4} \mathrm{~F}_{3}$ and $\mathrm{BC}_{4} \mathrm{~F}_{4}$ NILs were evaluated at Aurora NY for IP, LE, DLA, and DTA, following the previously described alpha design with B73 rows in every third row. The experiment was planted in two replicates, with five blocks per replicate, and four experimental rows plus two B73 rows per block.

To test whether the identified NLB QTL confer resistance to other important maize diseases, derived $\mathrm{BC}_{4} \mathrm{~F}_{3}$ and $\mathrm{BC}_{4} \mathrm{~F}_{4}$ NILs were evaluated at Aurora NY for anthracnose stalk rot (ASR) and common smut in 2007 and 2008, and for Stewart's wilt and common rust in 2008 and 2009. In 2007, the assessments of artificially inoculated ASR and naturally occurring common smut were conducted on the plants in NLB trial. In 2008 and 2009, derived NILs were grown in separate field plots for different disease evaluations. Trials for Stewart's wilt and common rust were planted following the previously described alpha design, with two replicates, five blocks per replicate, and four experimental rows plus two B73 rows per block. Trials for ASR were planted following the same alpha design, with two replicates, two blocks per replicate, and seven experimental rows plus four B73 rows per block. Plants in the trials of common smut were randomized in two replicates.

Four genotypes were used for microscopic analysis and DNA-based real time PCR quantification: B73, Tx303, TBBC3-38-05F (the NIL with $q N L B 1.06_{T \times 303}$ ), and TBBC3-42-10E-02 (the NIL with $q N L B 1.02_{T \times 303}$ ). Three greenhouse trials (each with five plants per genotype) and one field trial (five blocks, one row per genotype per block) were conducted from December 2007 to July 2008. Real-time PCR quantification was not performed in the 2008 field trial. In the greenhouse, plants subject to the same treatment were randomized within a block in order to eliminate the variance due to environmental factors.

\section{Data analysis}

Mixed model analyses were performed in JMP 7.0 for the field trails following an alpha design. For trials conducted in one field location, "maize lines" was specified as a fixed factor, whereas "replicates" and "blocks within replicates" were specified as random. Data from the 2006 trials in $\mathrm{NY}$ and NC were analyzed separately because of the different field arrangements and disease rating scales. A combined analysis across two locations (NY and NC) was conducted for the 2007 trials by fitting a mixed model with "maize lines", "locations" and "maize lines by locations" as fixed factors, and "replicates within locations" and "blocks within replicates within locations" as random effects. Phenotypic differences between TBBC3 lines/
NILs and B73, or between any two NILs, were determined by pair-wise comparisons of least squares means using two-tailed Student's t-test at $P<0.05$. Correction for multiple comparisons was not made since all the tests were independent. The TBBC3 lines and derived NILs were each compared to the recurrent parent B73, and pairs of NILs were only compared to each other when sharing identical genotypes except for the target introgression(s) under testing. Overall comparisons of TBBC3 lines or NILs were not intended in this analysis.

The QTL effect of each marker locus was investigated in the full TBBC3 population. Considering the presence of unlinked, potentially unrecognized introgressed segments in the same lines, a series of statistical tests was conducted as described by Szalma et al. [27] with some modification. Markers likely associated with resistance $(P$ $<0.05$ ) were identified by: 1) comparing the least squares mean of TBBC3 lines homozygous for Tx303 alleles at each locus to the least squares mean of $B 73$ rows, and 2) performing one-way analysis of variance (ANOVA) on an individual marker - trait basis in the TBBC3 population. Significant markers were grouped into linked introgression blocks, and the markers with the greatest significance in each linked block were considered to reflect the most likely QTL position. Correlations between the most significant markers were checked. For each pair of unlinked but correlated markers, TBBC3 lines fixed for one marker were grouped and analyzed for the co-segregation of the second marker and disease traits, using twotailed Student's $t$ test at $P<0.05$. A putative QTL was declared if the mean of all the lines with Tx303 alleles at this locus was significantly different from B73, and significant difference was detected for lines contrasting for this locus under the condition of another potential QTL locus being fixed.

In segregating populations, the phenotypic and genotypic data were first analyzed by mixed stepwise regression, with a significance probability of $P<0.05$ for each parameter to enter/leave the model [59]. Once the markers significantly associated with the traits were identified, ANOVA was conducted on a single marker basis at $P<$ 0.05 to estimate QTL effects. For the populations with more than one marker showing significant effect on traits, the effective markers were further tested for epistatic interactions by examining marker pairs by two-way ANOVA. Allele effects were determined by pair-wise two-tailed Student's t test according to the least significant difference (LSD) at $P=0.05$.

The proportion data from microscopic analysis and qPCR were transformed to arcsine of the square roots prior to statistical analysis. Arcsine transformed data were analyzed by fitting a linear least squares model with "genotype" and "replicate" as two independent variables, and with the number in the denominator of each propor- 


\section{Population of $\mathrm{BC}_{3}$ introgression lines}

(A) Phenotypic evaluation on the full set of 82 introgression lines

\section{(B) Identification of the outliers and putative QTL}

Subset of introgression lines

(C) Phenotypic evaluation on 15 selected introgression lines

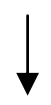

B73 $x$ selected introgression lines
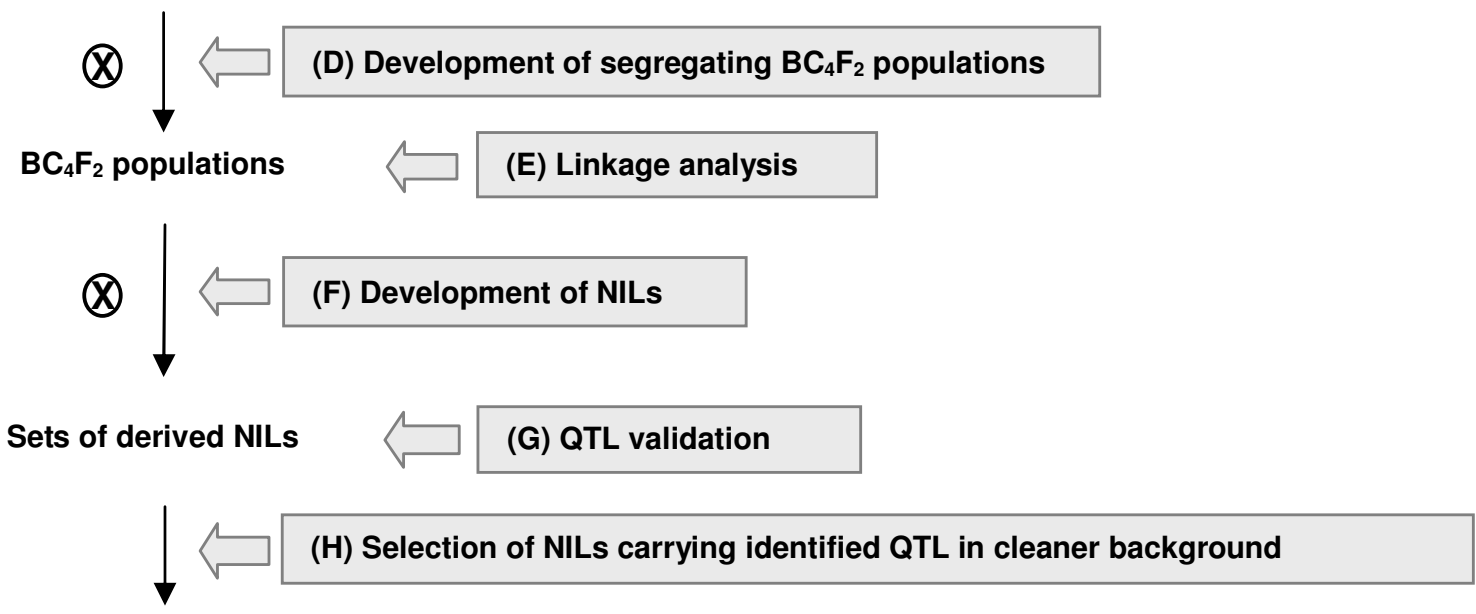

Selected NILs

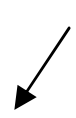

(I) Dissection of quantitative resistance to NLB
(J) Preliminary characterization of potential QTL effects on multiple diseases

Figure 1 Strategy for mapping and characterizing QTL using introgression lines. (A) Initial screening was conducted at Aurora NY and Clayton NC. Conventional disease components, including incubation period (IP), diseased leaf area (DLA), and disease severity were evaluated. (B) Lines that differed from B73, and lines showed extreme phenotypes in the population were determined. Putative QTL were also identified. (C) Lines carrying putative NLB QTL were evaluated to confirm their differential resistance/susceptibility relative to B73. Plants were tested at the juvenile and adult stages for IP, LE, and DLA. (D) Selected lines were backcrossed to $B 73$, then selfed to generate $F_{2}$ populations segregating for the introgressed regions within the populations. (E) Individual plants were tested for markers targeting introgressed regions, and co-segregation was assessed with the disease components measured in the greenhouse or field in NY. Candidate NLB QTL were determined if trait-marker association was detected in more than one $\mathrm{F}_{2}$ population. ( $\mathrm{F}$ ) The $\mathrm{BC}_{3} \mathrm{~F}_{3}$ and/or $\mathrm{BC}_{3} \mathrm{~F}_{4}$ near-isogenic lines (NILs) carrying different Tx303 introgressions were derived from $\mathrm{F}_{2}$ populations. (G) The effects of different introgressions on conventional disease components were further tested. The NLB QTL were declared if the QTL effects were validated in the NILS. (H) Selected NILs carrying identified QTL were used for detailed QTL characterization. (I) Selected NILs were evaluated with a series of disease components targeting different stages of disease development (Table 1) in the greenhouse and field. (J) Selected NILs were also evaluated for anthracnose stalk rot, common rust, common smut and Stewart's wilt at Aurora NY.

tion as a weighting factor. For qPCR, considering the biological and technical replicates, the fungal biomass ratios were also arcsine square-root transformed, but were analyzed with a different linear least squares model of $y_{\mathrm{ijm}}=$ $\beta_{0}+\beta_{1}\left(\right.$ genotype $\left._{\mathrm{i}}\right)+\beta_{2}\left(\right.$ rep $\left._{\mathrm{j}}\right)+\beta_{3}\left(\right.$ plate $\left._{\mathrm{m}}\left(\mathrm{rep}_{\mathrm{j}}\right)\right)+\mathrm{e}_{\mathrm{ijm}}$ (genotype $i=B 73$, Tx303 and two derived NILs; rep $j=1$, 2, 3; plate $\mathrm{m}=1,2,3 ; \mathrm{e}_{\mathrm{ijm}}$ : random experimental error).
Least squares means and 95\% confidence intervals were back-transformed to percentages. Confidence intervals were larger than significance levels due to asymmetry resulting from back transformation of arcsine scale. The significant QTL effects were estimated by pair-wise comparisons of least squares means of introgression lines/ NILs and B73 using two-tailed Student's t-test at $P<0.05$. 
Table 2: NLB resistance of the subset of 15 TBBC3 introgression lines.

\begin{tabular}{|c|c|c|c|c|c|c|c|}
\hline \multirow[t]{2}{*}{ Maize line } & \multirow[t]{2}{*}{$\begin{array}{l}\text { Introgressed } \\
\left(\text { Bin) }{ }^{a}\right.\end{array}$} & \multirow[t]{2}{*}{ Days to anthesis } & \multicolumn{3}{|c|}{$\begin{array}{l}\text { Trait (juvenile plants being inoculated; NY } \\
\text { and } N C)^{b}\end{array}$} & \multicolumn{2}{|c|}{$\begin{array}{l}\text { Trait (adult plants } \\
\text { being inoculated; NY) }\end{array}$} \\
\hline & & & $\begin{array}{l}\text { Relative } \\
\text { IP }\end{array}$ & $\begin{array}{l}\text { Relative } \\
\text { LE }\end{array}$ & $\begin{array}{l}\text { Relative } \\
\text { AUDPC }\end{array}$ & $\begin{array}{l}\text { Relative } \\
\text { IP }\end{array}$ & $\begin{array}{l}\text { Relatvie } \\
\text { AvgDLA }\end{array}$ \\
\hline Tx303 & & $8.7^{* * *}$ & $2.1^{* * *}$ & -0.3 & $-164.1^{* * *}$ & $7.5^{* * *}$ & $-8.7^{* * *}$ \\
\hline TBBC3-38 & $\begin{array}{c}1.03,1.06,5.00,5.03 \\
5.07-5.09\end{array}$ & $4.0^{* * *}$ & $2.1^{* * *}$ & -0.1 & $-110.2^{* * *}$ & $2.6^{* * *}$ & $-4.5^{* * *}$ \\
\hline TBBC3-39 & $1.03,1.06,5.00,5.07$ & $2.4^{* * *}$ & $0.9^{*}$ & 0.0 & $-67.2^{* *}$ & $1.3^{*}$ & $-4.3^{* * *}$ \\
\hline TBBC3-18 & $\begin{array}{c}1.11,7.05,7.06 \\
10.03,10.04\end{array}$ & $4.2^{* * *}$ & $0.8^{*}$ & -0.1 & -35.8 & $1.3^{*}$ & $-2.0^{* *}$ \\
\hline TBBC3-19c & $7.04,7.06,9.03,10.04$ & $1.4^{*}$ & $1.2^{* *}$ & 0.1 & -29.0 & 1.2 & $3.2^{* * * *}$ \\
\hline TBBC3-26 & 7.06 & 1.0 & $1.4^{* *}$ & 0.0 & -1.8 & -0.1 & 1.0 \\
\hline TBBC $3-75^{d}$ & $6.04-6.05$ & 1.2 & 0.4 & 0.1 & 12.2 & $2.6^{* * *}$ & $-2.3^{* *}$ \\
\hline TBBC3-02 & $\begin{array}{c}1.07-1.08,5.01-5.02 \\
8.03-8.05\end{array}$ & $3.2^{* * *}$ & -0.7 & 0.2 & 35.7 & 0.6 & $\underline{1.5}^{*}$ \\
\hline TBBC3-14c & $1.09-1.10,4.07$ & $3.1^{* * *}$ & $\underline{-0.9^{*}}$ & 0.0 & $\underline{67.0}^{* *}$ & 0.3 & $\underline{6.7}^{* * *}$ \\
\hline TBBC3-03 & $\begin{array}{c}1.01,1.04,7.03,8.03- \\
8.05\end{array}$ & $2.8^{* * *}$ & -0.1 & -0.1 & $\underline{117.0}^{* * *}$ & $-1.4^{*}$ & $\underline{5.7}^{* * *}$ \\
\hline TBBC3-61c & 1.10 & $1.4^{*}$ & -0.5 & $\underline{0.6}^{* * *}$ & $\underline{126.7}^{* * *}$ & 0.2 & $\underline{7.0}^{* * *}$ \\
\hline TBBC3-77 & $1.01-1.03,6.04-6.05$ & $4.3^{* * *}$ & $-1.4^{* *}$ & $\underline{0.9}^{* * *}$ & $139.4^{* * *}$ & $-1.4^{*}$ & $\underline{4.0}^{* * *}$ \\
\hline TBBC3-30 & $4.02-4.03,5.04$ & 0.2 & $\underline{-0.9^{*}}$ & $\underline{0.6}^{* * *}$ & $195.4^{* * *}$ & -0.6 & $4.7^{* * *}$ \\
\hline TBBC3-21 & $4,07,9.01-9.04$ & 0.6 & $-1.7^{* * *}$ & $\underline{0.8}^{* * *}$ & $\underline{275.5}^{* * *}$ & -1.1 & $\underline{7.8}^{* * *}$ \\
\hline TBBC3-36 & $\begin{array}{c}1.01,1.03-1.05,4.01 \\
8.08\end{array}$ & 1.3 & -0.8 & $\underline{0.7}^{* * *}$ & $\underline{305.6}^{* * *}$ & ${\underline{-1.8^{* *}}}^{*}$ & $\underline{8.2^{* * *}}$ \\
\hline TBBC3-42 & $\begin{array}{l}1.01-1.02,4.07-4.08 \\
5.01-5.02,8.02-8.05\end{array}$ & $2.2^{* *}$ & $\underline{-2.1}^{* * *}$ & $\underline{0.7}^{* *}$ & $\underline{310.8}^{* * *}$ & $-1.3^{*}$ & $\underline{8.7}^{* * *}$ \\
\hline
\end{tabular}

In 2007, the same set of plant materials was inoculated at the five- to six-leaf stage in New York (NY) and North Carolina (NC), and were grown in a separate field plot in NY and inoculated 1-2 weeks before anthesis. Trait values shown are the least squares means of maize lines relative to the recurrent parent B73. Phenotypes that were significantly more resistant than B73 are highlighted in bold, while the phenotypes significantly more susceptible than B73 are underscored. The significance level was determined by pair-wise two-tailed Student's t test on the least square difference (denoted as ${ }^{*} 0.01<P<0.05 ;{ }^{* *} 0.001<P<0.01{ }^{* * *} P<0.001$ ). Maize lines in bold are the ones that were consistently more resistant or more susceptible than $B 73$ across two field environments.

a Introgressed genome regions from Tx303 (heterozygous regions not shown). Bin position was based on genetic map of intermated B73 $\times$ Mo17 population (version IBM 2008 neighbors).

b Relative least squares means of incubation period (IP) and area under the disease progress curve (AUDPC) shown were from the combined analysis across the trials in NY and NC. Lesion expansion (LE) was only scored in NY.

c Individual TBBC3 lines in each field environment were inspected. TBBC3-19 was significantly more resistant than B73 for IP $(P=0.03)$, DLA1 $(P=0.001), \mathrm{DLA} 2(P=0.035)$ and AUDPC $(P=0.010)$ only in NC. TBBC3-14 and TBBC3-61 showed greater susceptibility than B73 for three DLA ratings $(P<0.003)$ and AUDPC $(P<0.0001)$ only in NY. The same differential degrees of resistance or susceptibility in two field environments were also observed in 2006 (Additional file 1).

d Resistance to NLB was more effective in adult plants.

All the statistical analyses in the study were conducted using JMP 7.0.

\section{Results}

A stepwise strategy (Figure 1) was conducted to map and characterize QTL using the TBBC3 introgression lines and derived NILs.
Identification of outliers and putative NLB QTL in the TBBC3 population

The full set of 82 TBBC3 lines was screened for NLB resistance at field sites in New York and North Carolina in 2006. As shown in Additional file 1, the TBBC3 population was generally more susceptible than B73 at both locations. In the trial at Aurora NY, three lines were 
scored as significantly more resistant and 20 lines were scored as significantly more susceptible than B73 for primary DLA, while no lines showed significantly more resistance and 39 lines showed more susceptibility than B73 based on AUDPC. In the trial at Clayton NC, eight lines were significantly more resistant and 13 lines were significantly more susceptible than B73 for AUDPC. The only line that showed contrasting phenotypes in the two environments was TBBC3-25, suggesting experimental error or environmental influence on the expression of NLB QTL.

A combination of analytic approaches was used to associate the resistance or susceptibility effects with specific chromosomal segments [27]. Based on this analysis, 13 putative NLB QTL were identified in the TBBC3 population (Additional file 2). Except for bins 1.01 and 5.08-5.09, the putative QTL co-localized with NLB QTL previously detected in four maize mapping populations [20].

\section{Phenotypic evaluation on the subset of 15 TBBC 3 lines}

A subset of 15 TBBC3 lines was selected for further evaluation based on the degree of difference in NLB resistance between each individual TBBC3 line and B73 at the two field sites (Additional file 1 and Table 2). Lines significantly or marginally different $(P<0.15)$ from B73 in either of the two locations were taken into consideration. Line selection was also influenced by the identity of the Tx303 introgressions carried by the lines; those carrying introgressions corresponding both to putative QTL identified in the TBBC3 population (Additional file 2), and to previously reported NLB QTL (specifically those at bins 1.07-1.08, 5.02, and 6.04-6.05 [20]) were given priority because of our interest in QTL validation and characterization rather than in QTL discovery.

In 2007, the 15 selected lines were evaluated at the same field locations in NY and NC to confirm their disease phenotypes. Significant "line" effects $(P<0.0001)$ were found for all disease parameters in each of NY and $\mathrm{NC}$, and across two locations. In the combined analysis across environments, significant effects of "location" $(P<$ 0.002 for IP, DLA and AUDPC) and "line-by-location" ( $P$ $<0.0001$ for DLA and AUDPC) were also detected. The variations were attributed to dissimilar pathogen strains, environmental conditions and disease ratings conducted by different scorers at two sites. Inspection of individual TBBC3 lines in NY and $\mathrm{NC}$ respectively suggested that most of the lines performed similarly across the two locations. Pair-wise comparisons validated the phenotypic differences for seven out of the 15 TBBC3 lines and B73 (Table 2). Consistently in NY and NC, TBBC3-38 and TBBC3-39 were more resistant, whereas TBBC3-42, ТВВС3-36, ТВВС3-21, ТВВС3-30, and ТВВС3-77 were more susceptible than $\mathrm{B} 73$.
Ten selected TBBC3 lines showed significantly greater DTA than B73 (about 1-4 days of difference, Table 2), indicating the presence of flowering time QTL in those lines. To test the effect of plant development on QTL expression, the 15 selected lines were inoculated at juvenile and adult stages (Table 2) and their disease traits were compared. In all the lines, QTL effects in reducing or increasing resistance to NLB were generally consistent for juvenile and adult plant stages. No lines showed contrasting phenotypes (more resistant and more susceptible relative to B73) when inoculated at the five- to six-leaf stage or just before flowering, which suggested that allele effects of NLB QTL were not altered by developmental stage. There was, however, evidence suggesting that the effectiveness of QTL might change over plant development. The resistance in TBBC3-38, ТВBC3-39, TBBC318 and TBBC3-75 was more effective in adult than in juvenile individuals. The formation of NLB lesions was delayed by around 0.5-2 days on mature plants of the same genotypes.

\section{Validation of QTL effects by linkage analysis}

Each of the TBBC3 lines had more than one identified introgression from Tx303. To determine which of the introgressed regions in a given line was associated with resistance, several segregating $\mathrm{BC}_{4} \mathrm{~F}_{2}$ populations were developed from crosses between selected ТВBC3 lines (ТВBC3-02, ТВВC3-38, ТВВC3-39, ТВВC3-42, and TBBC3-77) and the recurrent parent $\mathrm{B} 73$. The $\mathrm{BC}_{4} \mathrm{~F}_{2}$ individuals from each cross were evaluated for NLB resistance and genotyped using markers for the respective introgressed regions. Significant trait-marker associations were identified in all the $\mathrm{BC}_{4} \mathrm{~F}_{2}$ populations under investigation (Additional file 3).

The QTL at bin 1.02 was found effective in two segregating populations: those derived from TBBC3-42 and TBBC3-77. Although the introgressed region extended from bin 1.01-1.02, higher significance was detected at the marker located at bin 1.02 so the QTL was designated qNLB1.02. In the $\mathrm{BC}_{4} \mathrm{~F}_{2}$ derived from TBBC3-42, 14\% of the IP variation could be attributed to $q N L B 1.02$. The B73 allele at $q N L B 1.02$ was associated with slower disease development. Individuals homozygous for Tx303 alleles in bin 1.02 (replacement of $q N L B 1.02_{B 73}$ ) were significantly more susceptible than B73. The Tx303 homozygotes showed lesions $\sim 0.6$ days earlier (lower IP; $P<$ 0.0001 ) and lesions expanded more rapidly by $\sim 0.35 \mathrm{~mm} /$ day (greater LE; $P<0.05$ ) in the B73 $\times$ TBBC3-42 population. The same QTL acted differently in the B73 $\times$ TBBC3-77 population. While qNLB1.02 was effective in reducing DLA and AUDPC, no effect on IP was observed in the B73 $\times$ TBBC3-77 population. It is worth noting that TBBC3-42 is a much more susceptible genotype than 
TBBC3-77, which provided a better background for evaluating minor disease QTL.

The QTL at bin 1.06 was not identified based on previously reported genotype information. Although clear phenotypic variation was observed on IP and DLA in the $\mathrm{BC}_{4} \mathrm{~F}_{2}$ populations from TBBC3-38 and TBBC3-39, no significant association was found between IP and the known introgressions at bins 1.03, 5.00, 5.01-5.03, and 5.07-5.09. The IP on $\mathrm{BC}_{4} \mathrm{~F}_{2}$ individuals of TBBC3-38 and TBBC3-39 ranged from 11 to $18 \mathrm{dpi}$, while IP on B73 was generally between 12 and $15 \mathrm{dpi}$. Since these two populations displayed a greater variation in resistance, we inferred that a disease QTL was present in the population but not associated with a recognized introgression. To search for unrecognized introgressions, an additional 68 SSR markers across the genome were chosen to target chromosomal regions that were not well-covered by the original 130 RFLP and SSR markers on TBBC3 map. Pooled DNA samples from $\mathrm{BC}_{4} \mathrm{~F}_{2}$ individuals of TBBC338 and TBBC3-39, along with B73 DNA as a control, were tested with the additional SSR markers for heterozygosity. Among the 68 SSR markers tested, umc1754 and umc2234 (both in bin 1.06) were the only polymorphic markers segregating in both $\mathrm{BC}_{4} \mathrm{~F}_{2}$ populations. This indicated the presence of a Tx303 introgression at bin 1.06 in both TBBC3-38 and TBC3-39. Individuals in each population were then genotyped with umc1754 and umc 2234, and analyzed for the effects on various disease components. A highly significant association was detected for various parameters in both populations (Additional file 3). In the B73 $\times$ TBBC3-38 and B73 $\times$ TBBC3-39 populations, around $33 \%$ and 53\% (respectively) of the variation in AUDPC was explained by this QTL. Compared to the B73 allele, the Tx303 allele at $q N L B 1.06$ increased the IP by $\sim 2$ days, decreased LE by $\sim 0.3 \mathrm{~mm}$ per day, and reduced DLA by about $5-10 \%$. Another QTL at bins 5.08-5.09 (Tx303 allele for resistance) was found in the $\mathrm{B} 73 \times \mathrm{TBBC} 3-38$ population. The effect was relatively minor $\left(R^{2} \approx 0.05\right)$ compared to the $q N L B 1.06_{T \times 303}$ identified in the same population.

QTL were also identified in bins 5.00, 5.01-5.02, and 8.03-8.05. Although contributing significant levels of resistance for IP or DLA in the B73 $\times$ TBBC3-77 and B73 $\times$ TBBC3-02 populations, the same introgressions/markers were not found effective in the populations derived from ТВBC3-38, ТВBC3-39 and ТВBC3-42. The ambiguous results called the association into question, suggesting that the effectiveness of QTL at bins 5.00, 5.01-5.02, and 8.03-8.05 was affected by genetic background and/or environmental conditions.

Validation of $q N L B 1.02_{B 73}$ and $q N L B 1.06_{T \times 303}$ in selected NIL sets

To confirm the QTL effects detected in segregating populations, NILs were generated by selfing selected $\mathrm{BC}_{4} \mathrm{~F}_{2}$ lines from the populations $\mathrm{B} 73 \times \mathrm{TBBC} 3-38, \mathrm{~B} 73 \times$ TBBC3-39 and B73 $\times$ TBBC3-42. Selected lines were chosen to represent different introgressed regions in the original TBBC3 lines. The QTL effects were determined by pair-wise comparisons between individual introgression lines and B73, and between the NILs developed from a TBBC3 line. Within each set of NILs, lines contrasting for each marker locus were grouped and analyzed for their phenotypic differences. The NILs derived from different TBBC3 lines were not compared to each other since the phenotypic difference can be attributed to not only introgressed regions but also to minor differences in genetic backgrounds.

Evaluations of the $\mathrm{BC}_{4} \mathrm{~F}_{3}$ NILs validated the effects of qNLB1.06 $6_{T \times 303}$. Five NILs derived from B73 $\times$ TBBC3-38 and four NILs derived from B73 $\times$ TBBC3-39 were phenotyped in 2008. Individual trait-locus analysis in the two NIL sets revealed that bin 1.06 was the only locus that showed significant effects on resistance. The QTL effect at bin 5.08-5.09 was detected in the B73 $\times$ TBBC3-38 population, but was not confirmed in the NILs. As shown in Additional file 4, most of the NILs carrying Tx303 alleles at bin 1.06 were significantly more resistant than B73. The only exception was the line TBBC3-39-11A, which might have lost the resistance gene(s) due to recombination. Significant differences between lines with $q N L B 1.06_{T \times 303}$ and B73 were observed for IP, DLA and AUDPC, which was in agreement with the results from linkage analysis in $\mathrm{BC}_{4} \mathrm{~F}_{2}$ populations (Additional file 3). The effects of $q N L B 1.06_{T \times 303}$ relative to $q N L B 1.06_{B 73}$ on IP (1.5-2.5 days) and DLA (2-10\% through the season) were determined by comparing TBBC3-38-05F with B73 (Figure 2A) and TBBC3-39-19E with B73. The significant effect of reducing lesion expansion was not confirmed in the NILs. Lines with $q N L B 1.06_{T \times 303}$ even showed significantly higher LE scores, indicating some variations from different genetic backgrounds and environments.

qNLB1.02 was validated by analyzing six $\mathrm{BC}_{4} \mathrm{~F}_{3}$ NILs and seven $\mathrm{BC}_{4} \mathrm{~F}_{4}$ NILs derived from a B73 $\times$ TBBC3-42 cross. Evaluations conducted in 2007 and 2008 led to similar results. Since $\mathrm{BC}_{4} \mathrm{~F}_{4}$ NILs had cleaner genetic backgrounds, the data from the 2008 trial was shown to represent the overall result. Individual trait-locus analysis in this NIL set suggested that bin 1.01-1.02 was the only locus affecting resistance. The NILs carrying Tx303 allele(s) at bin 1.01-1.02 were all significantly more susceptible than the ones carrying B73 allele(s) at the same region (Additional file 5), confirming the resistance effect of $q N L B 1.02_{B 73}$. Highly significant differences between TBBC3-42-10E-02 (the NIL with $q N L B 1.02_{T \times 303}$ in the cleaner background) and $\mathrm{B} 73$ were observed across all the disease components under investigation (Figure 2). The B73 allele(s) at qNLB1.02 delayed lesion formation by 1.6 days $(P=0.004)$, inhibited lesion expansion by $0.84 \mathrm{~mm}$ 


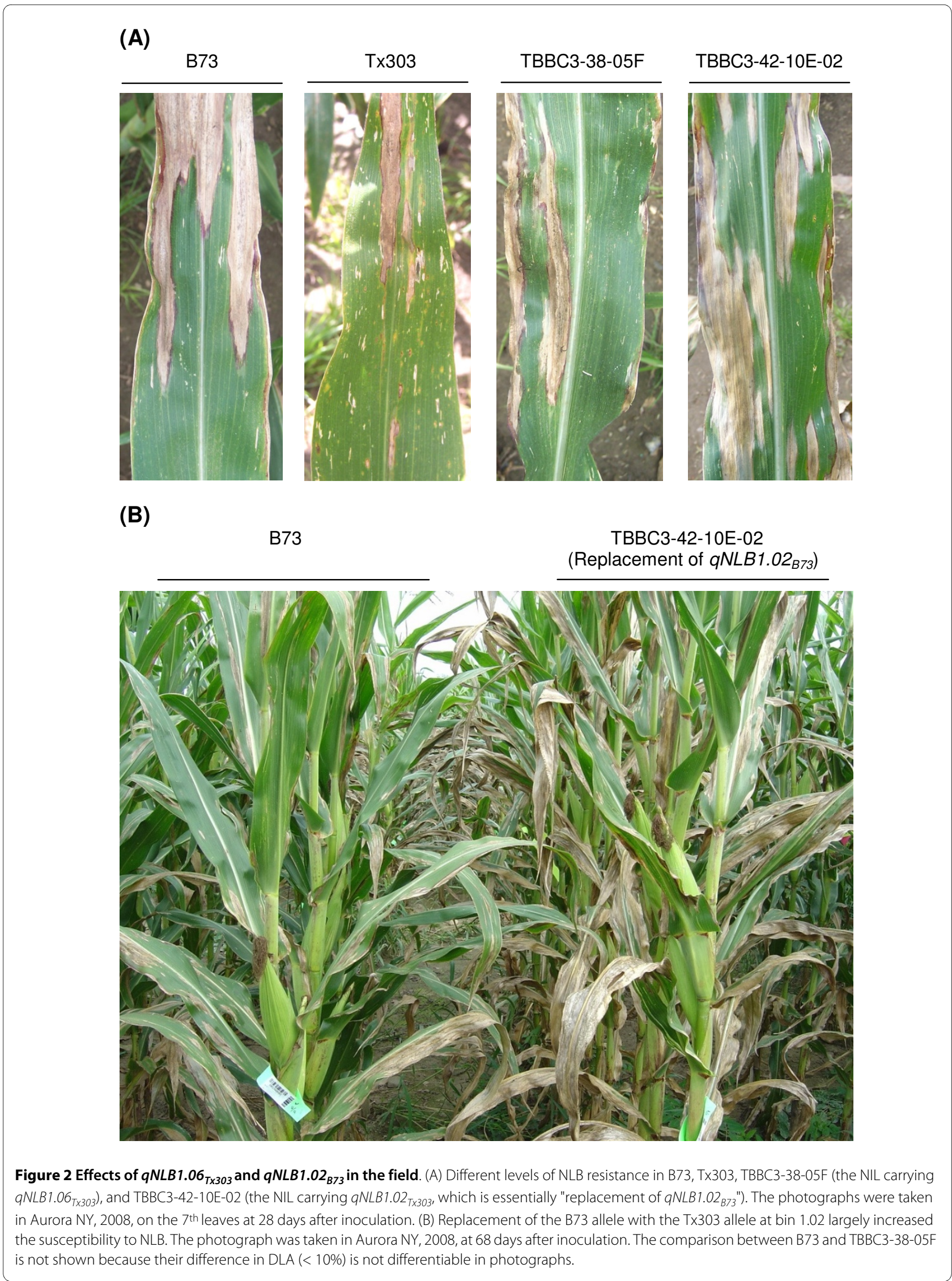


per day $(P<0.0001)$, and reduced DLA $18-38 \%$ through the season $(P<0.0001)$. Overall, the relative allele effects detected in the NILs were much greater than in the segregating populations, and the resistance of $q N L B 1.02_{B 73}$ was more effective in the field than in the greenhouse.

\section{Characterization of $q N L B 1.02_{B 73}$ and $q N L B 1.06_{T \times 303}$ using derived NILs}

Two derived NILs carrying identified NLB QTL in "cleaner" B73 backgrounds (minimal amount of introgression from Tx303 not associated with NLB resistance) were chosen for detailed QTL characterization. TBBC338-05F was a $\mathrm{BC}_{4} \mathrm{~F}_{3}$ NIL with a Tx303 introgression at bin 1.06 (designated as the NIL with $q N L B 1.06_{T \times 303}$ ), and TBBC3-42-10E-02 was a $\mathrm{BC}_{4} \mathrm{~F}_{4}$ NIL with a Tx303 introgression at bin 1.02 (designated as the NIL with $\left.q N L B 1.02_{\text {Tx303 }}\right)$. To investigate the effect of QTL during NLB development, a series of disease components (Table 1) was selected or developed and applied on the two derived NILs in three replicated experiments in the greenhouse during December 2007 - April 2008, and at Aurora, NY during summer 2008.

\section{Microscopic investigation on the pathogenesis of S. turcica}

Trypan blue staining and $\mathrm{KOH}$-aniline blue fluorescence microscopy techniques were used for histological examination of NLB development in planta. To understand typical resistant and susceptible interaction patterns in the NLB pathosystem, preliminary microscopic analysis was conducted on the following maize lines challenged with S. turcica: B73, Tx303, TBBC3-38, TBBC3-42, the resistant maize inbred line CML52 and CML103, and a susceptible recombinant inbred line IBM262. As illustrated in Figures 3 and 4, pathogenesis was successfully visualized and can be summarized as follows. After landing and attaching on the leaf surface of a susceptible maize plant, the conidium of $S$. turcica germinates. It forms an appressorium, which produces a penetration peg that punches through the cell wall and into the epidermal cell. From this cell, infective hyphae are produced, grow towards the vascular bundle, enter the vasculature and ramify within it. Aggressive hyphal growth and extension in the vascular bundles can be seen. The hyphae can grow for several days inside the vascular bundles without causing visible symptoms (incubation period was approximately seven days on susceptible maize lines). After extensive growth within the vascular veins, hyphae grow out to colonize the neighboring bundle sheath cells and then branch out to colonize the rest of leaves. This progression is consistent with several previous studies on S. turcica pathogenesis on maize [23-25]. The limited damage $S$. turcica causes to host tissues at early phase of pathogenesis and the extended incubation period suggests the possibility that this is a hemibiotrophic interaction.
Among diverse maize genotypes (B73, Tx303, CML52, CML103 and IBM262) and on genotypes carrying different QTL for resistance (B73, TBBC3-38, and TBBC3-42), differential phenotypes were observed for the timing and extent of certain steps in the processes of infection and colonization. To document these differences, a series of microscopic parameters was chosen for characterizing NLB QTL efficacy at different stages during pathogenesis. The microscopic disease components used in the study included the number of appressoria, infection efficiency, vascular invasion efficiency, the size of strongly fluorescent area surrounding the infection site, and the appearance of necrotic or fortified vascular bundles. The microscopic evaluations on B73, Tx303 and the derived NILs were performed on plants grown in controlled greenhouse conditions as well as in the field.

\section{QTL effect on the infection of S. turcica}

Infection efficiency was examined by trypan blue and $\mathrm{KOH}$-aniline blue staining, followed by scoring the number of conidia that had successfully penetrated the epidermal cell walls. The infection efficiency was defined as the ratio of successful infection sites over the total number of germinated conidia. Non-germinated conidia were excluded because of the difficulty of determining whether a non-germinated conidium was viable, given the fact that a small proportion of damaged conidia was always present in the spore suspension. In both the greenhouse and field, lower infection efficiency was consistently observed on the NIL with $q N L B 1.06_{T \times 303}$ at earlier time points examined, as well as on Tx303 at all time points examined (Figure 5A), compared with B73. In the greenhouse, $q N L B 1.06_{T x 303}$ reduced infection efficiency by $\sim 14 \%$ at $2 \mathrm{dpi}$ and by $\sim 24 \%$ at $4 \mathrm{dpi}(P<0.0001)$. Similarly, in the field, infection efficiency was reduced by $\sim 12 \%$ at 3 dpi $(P<0.0001)$ relative to $\mathrm{B} 73$. The resistant parental genotype Tx303 showed a stronger effect on reducing the infection of S. turcica in the field (decreased by $\sim 19 \%$ relative to B73, $P<0.0001)$ than in the greenhouse (decreased by $6.5-12.5 \%$ relative to $\mathrm{B} 73, P<0.05$ ). No significant effect on pathogen penetration was seen from qNLB1.02 ${ }_{\text {Tx303. }}$.

The incidence of multiple appressoria per germinated conidium was used as another indicator for resistance to fungal penetration. It was observed that when a germinated conidium failed to penetrate the epidermis, the conidium would continue to produce new germ tubes and appressoria until exhausting its reserves or ultimately gaining entry into a plant cell (Figure 5B). Despite the effect of $q N L B 1.06_{T \times 303}$ on reducing the penetration of $S$. turcica, no difference was detected on the formation of multiple appressoria among B73, Tx303, and the derived NILs. 

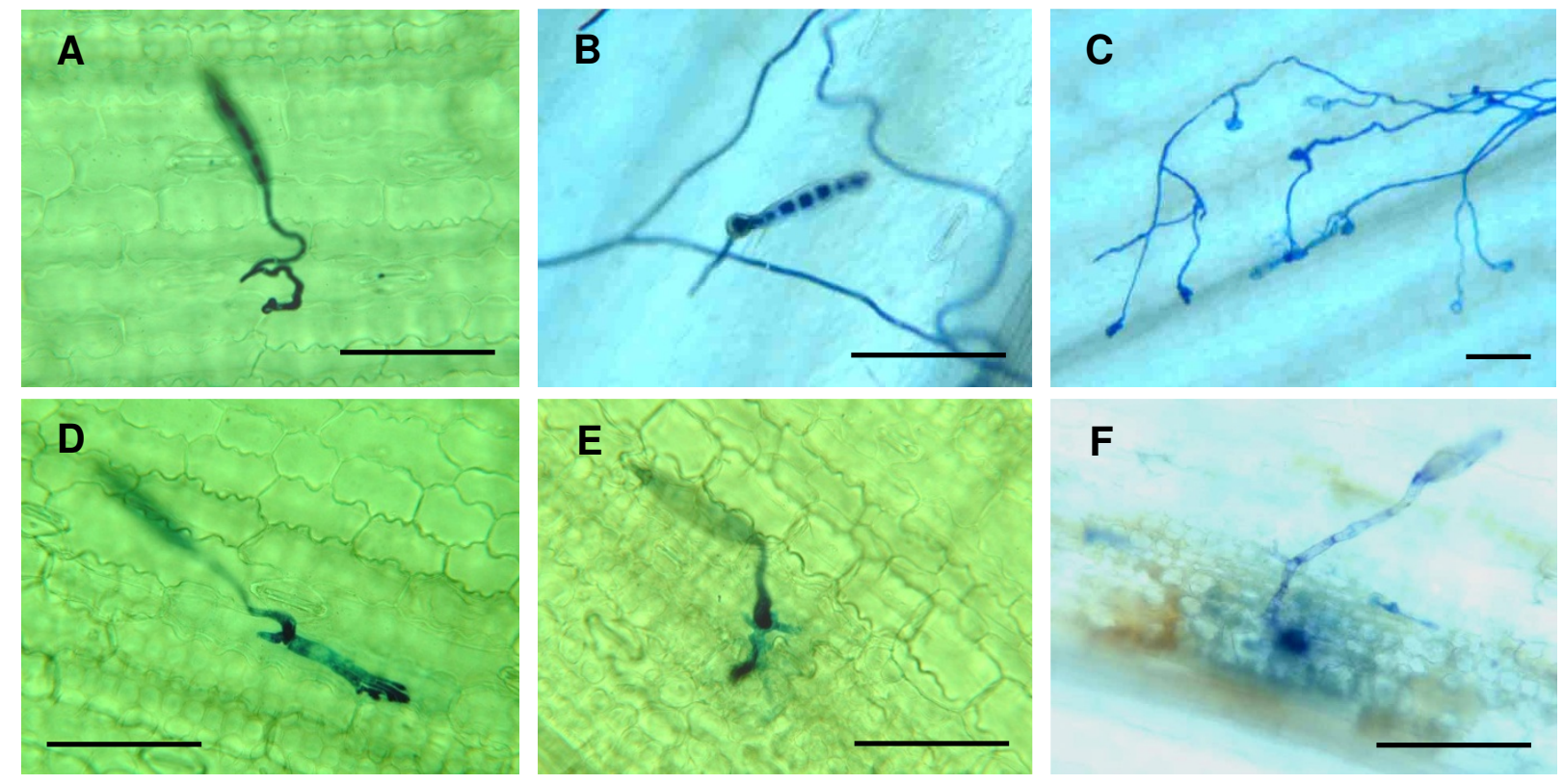

Figure 3 Light micrographs of the infection and early colonization of Setosphaeria turcica in corn leaves. Samples from greenhouse trials were stained with trypan blue. (A) A conidium germinated and formed an appressorium. (B) The penetration peg from the appressorium punched through the cuticle and epidermal cell wall. (C) The conidium could continue to produce new germ tubes and appressoria until exhausting its reserves or ultimately gaining entry into a plant cell. This phenomenon was occasionally observed on a resistant inbred line CML52. (D) A subcuticular palm-shaped structure occasionally developed from the penetration peg (likely before hyphae infected the epidermal cell). (E and F) Cytoplasmic depletion of the conidium was seen after the infection process. Infective hyphae spread from primary infected cell to surrounding area, causing host cell death. (Infected leaf samples of A, D and E: CML52, 2dpi; B: B73, 2 dpi; C: CML52, 3 dpi; F: IBM262, 5 dpi) (Scale bars, 100 ㅆm)

\section{QTL effect on the vascular invasion of S. turcica}

Intracellular hyphal growth from the initially infected epidermal cell to surrounding mesophyll cells, and the subsequent invasion of the vascular bundle, were investigated by $\mathrm{KOH}$-aniline blue fluorescence microscopy. Vascular invasion efficiency was defined as the incidence of hyphal growth into the vasculature per infection site. Differences in vascular invasion efficiency were observed between B73 and the other genotypes (Figure $5 \mathrm{C}$ ). The NIL with $q N L B 1.02_{T \times 303}$ had greater vascular invasion efficiency, indicating the superiority of the B73 allele(s) at this locus. Replacement of the B73 allele with the Tx303 allele at $q N L B 1.02$ (in lines carrying an introgression from Tx303 at this locus) led to $15-24 \%$ of increase in the incidence of vascular invasion in both greenhouse and field $(P<0.0001)$. The $q N L B 1.06_{T \times 303}$ allele(s) was not effective for inhibiting hyphal growth into the vasculature. Under greenhouse conditions, the NIL with $q N L B 1.06_{T \times 303}$ was observed to have a $7-19 \%$ higher susceptibility for vascular invasion $(P<0.0001)$, which is a further indication that this QTL functions at the stage of penetration but not at later stages of pathogenesis. The susceptibility to vascular invasion was also seen in Tx303. In the greenhouse, higher vascular invasion efficiencies were observed in Tx303 than in B73, although with mar- ginal significance levels. In the field, the invasion efficiency in Tx303 was $\sim 22 \%$ greater than in B73 $(P<$ 0.0001).

\section{QTL effects on the accumulation of defense compounds around the infection site}

Infected tissues stained with aniline blue were also used for revealing defense responses induced upon pathogen challenge. The fluorescence emitted from the cells surrounding the infection site is presumably due to callose deposition $[60,61]$ and the accumulation of autofluorescent phenolic compounds [62-64]. Callose and phenolics are antimicrobial compounds that help restrict the establishment and spread of S. turcica in the leaf. In susceptible genotypes, weak fluorescence and collapsed cells were generally observed around the infected sites (Figure 4I), suggesting the lack of induced defense responses. The more resistant genotypes showed enhanced fluorescence from localized cell regions (Figure $4 \mathrm{C}$ and $4 \mathrm{H}$ ).

To assess the degree of defense response around the primary infected cell, the diameter of brightly fluorescing area was measured under a fluorescence microscope with a micrometer (Figure 5D). The average diameter of the fluorescing area in the NIL with $q N L B 1.02_{T \times 303}$ was consistently smaller than that seen for B73 $(P<0.0001$ in greenhouse, $P=0.02$ in the field), indicating that the B73 

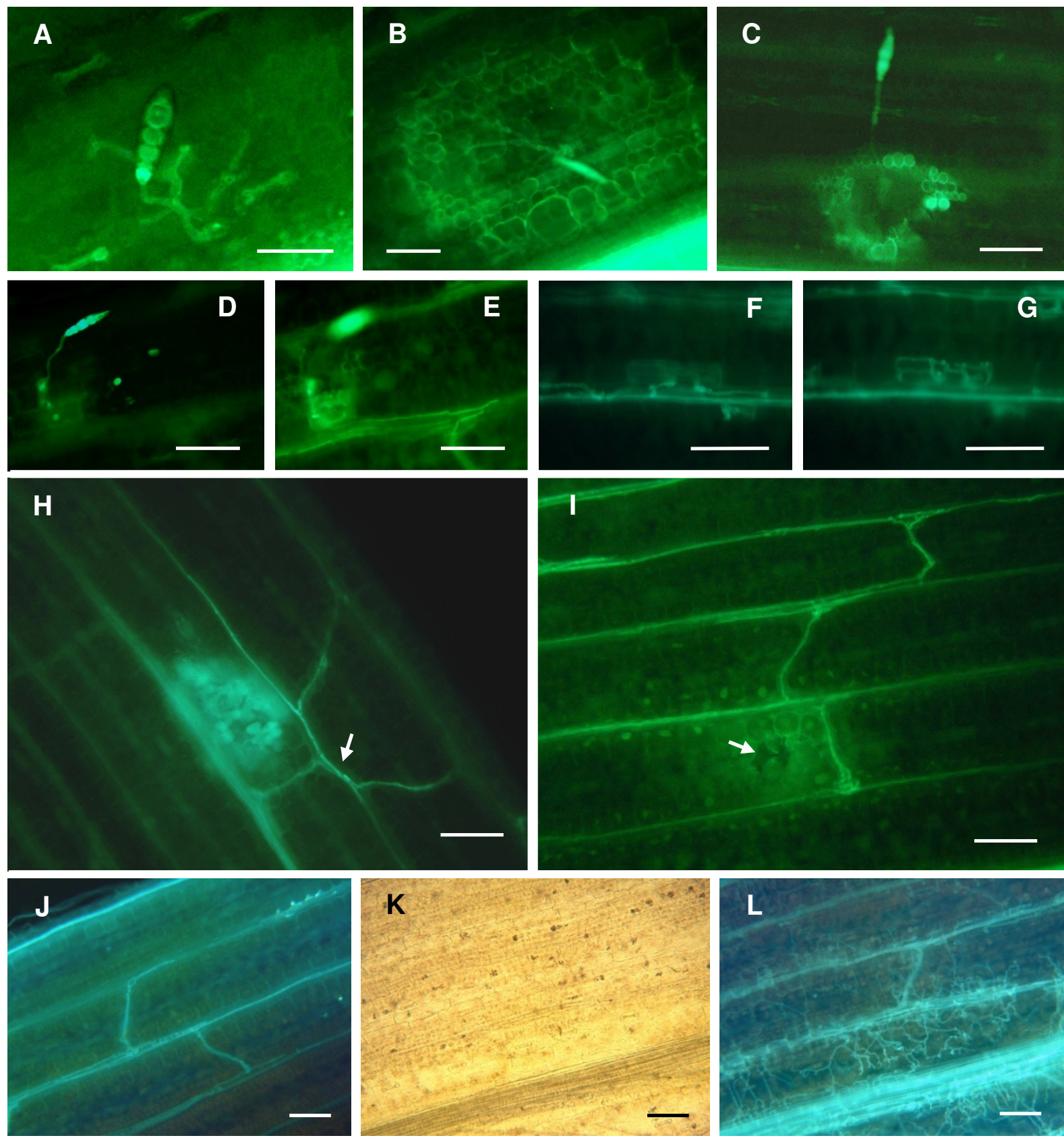

Figure 4 Fluorescence micrographs of the pathogenesis of Setosphaeria turcica in corn leaves. Samples from greenhouse trials were treated with $\mathrm{KOH}$-aniline blue. (A) A germinated conidium on leaf surface. (B) Infective hyphae grew into contact with mesophyll cells. (C and H) Defense responses induced around the infection site. The brightly fluorescing area was presumably caused by callose deposition and the accumulation of autofluorescent phenolic compounds. The fluorescing vascular bundles (arrow), possibly due to lignification, could be differentiated from the hyphae growing in the vasculature by the lack of distinguished hyphal coils. ( $D$ and $E$ ) Infective hyphae grew towards the vascular bundle and invaded it. $D$ and $E$ represent different focal planes. ( $F$ and $G$ ) Hyphae grew out the vascular vessel to colonize the neighboring bundle sheath cells. $F$ and $G$ represent different focal planes. (I) Hyphae successfully spread through the vascular system. The weak fluorescence and collapsed cells surrounding the infection site (arrow) were typical symptoms of the compatible interaction. (J) Movement of infective hyphae through vascular bundles and cross veins. Leaf tissue remained non-wilted at this stage. (K and L) Colonization of necrotic hyphae in the wilted lesion. Vascular bundles were plugged with aggressively growing hyphae. Hyphae branched out from the colonized vasculature to the rest of the leaf. $K$ and $L$ were viewed in the light- and fluorescent-field, respectively. (Infected leaf samples of A: Tx303, 4 dpi; B: CML52, 4 dpi; C: B73, 4 dpi; D and E: TBBC3-42, 4 dpi; F and G: CML103, 7 dpi; H: B73, 4 dpi; I to L: B73, 10 dpi) (Scale bars, $100 \mu \mathrm{m}$ ) 
(A)

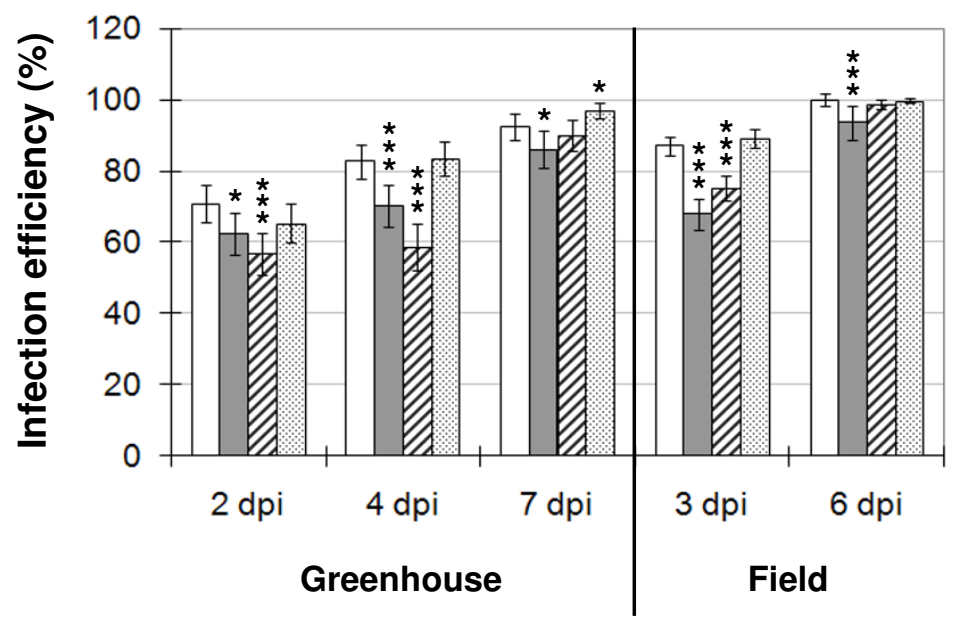

(B)

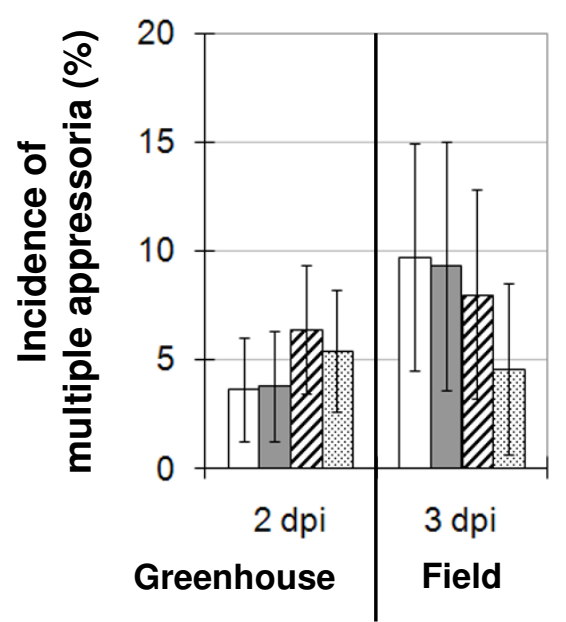

(C)

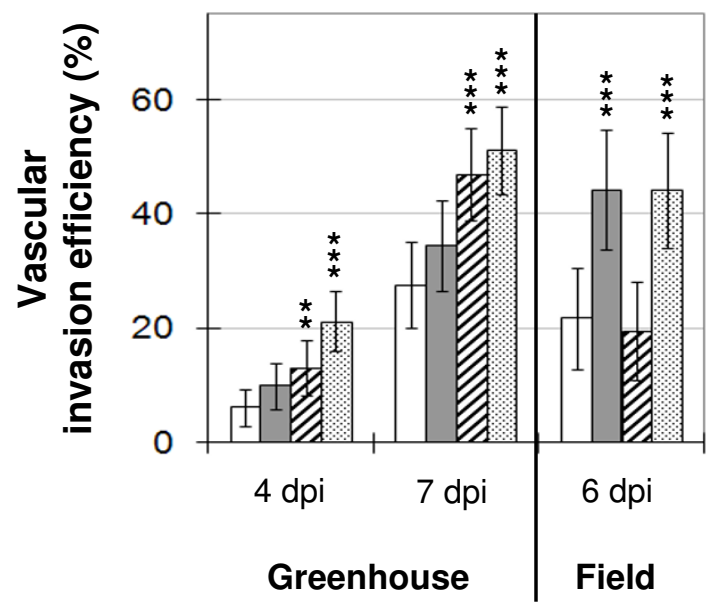

B73 $\square$ Tx303

(D)

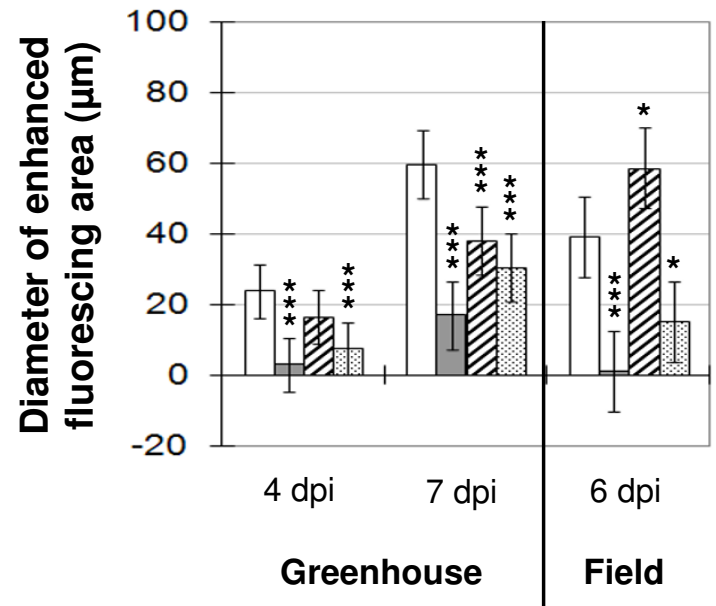

TBBC3-42-10E-02

(Replacement of qNLB1.02 ${ }_{B 73}$ )

Figure 5 Investigation of QTL effects on microscopic disease components. Microscopic disease components including (A) Infection efficiency, (B) incidence of multiple appressoria, (C) vascular invasion efficiency, and (D) size of strongly fluorescing area surrounding the infection site, were used to assess QTL effects in controlled greenhouse condition and field. Infected leaf samples were collected from maize genotypes B73 (open), Tx303 (gray), TBBC3-38-05F (hatched; the NIL carrying 9NLB1.06 Tx303) and TBBC3-42-10E-02 (dotted; the NIL carrying 9NLB1.02 Tx303, which is essentially "replacement of $q N L B 1.02_{B 73}$ "). Samples collected 2 days post inoculation (dpi) from greenhouse and 3 dpi from field were stained with trypan blue, while the samples collected $4 \mathrm{dpi}$ and $7 \mathrm{dpi}$ from greenhouse and $6 \mathrm{dpi}$ from field were treated with $\mathrm{KOH}$-aniline blue fluorescence technique. Differences between least square means of different genotypes relative to $B 73$ were determined by two-tailed Student's $t$ test (significance level: ${ }^{*} 0.01<P<0.05$; $\left.{ }^{* *} 0.001<P<0.01 ;{ }^{* * *} P<0.001\right)$. In graphs A - C, the proportion data were arcsine transformed for statistical analysis, and the corresponding least squares means and $95 \%$ confidence intervals were back transformed to original scale before plotting. The confidence intervals are bigger than significance levels due to asymmetry resulting from back transformation.

allele(s) at $q N L B 1.02$ contribute to the regulation, production or accumulation of callose, lignin or other phenolic compounds. Interestingly, compared to B73, the fluorescing area in the NIL with $q N L B 1.06_{T \times 303}$ was slightly larger $(P=0.052)$ in the field, but was significantly smaller $(P<0.0001)$ at $7 \mathrm{dpi}$ in the greenhouse, suggesting that the phenotype conferred by $q N L B 1.06_{T \times 303}$ is vulnerable to environmental influences. 
Considering the potential role of vascular resistance in the NLB pathosystem, the incidence of brightly fluorescing vascular bundles (likely caused by lignification) was also evaluated. Although this response was pronounced in CML52 (Figure 4H), this type of resistance was rarely observed in B73, Tx303 and the derived NILs. The induced defense reaction around the infection site was fairly weak at all times examined in either greenhouse or field conditions. It was thus not considered a relevant parameter for testing the effect of $q N L B 1.06_{T \times 303}$ and $q N L B 1.02_{B 73}$.

\section{QTL effect on mycelial growth of S. turcica in planta}

DNA-based qPCR was developed to precisely quantify mycelial growth of $S$. turcica in maize leaves. The $R$ square values for the two standard curves based on B73 and Tx303 respectively were both higher than 0.99 (Figure 6A), indicating that the designed ITS primer pair had good sensitivity and specificity for reliably amplifying fungal DNA. To assess the qPCR technique as a tool for analyzing the NLB pathosystem, several time-course experiments were performed on seven maize genotypes with a wide range of differential levels of NLB resistance (C. Chung, unpublished). Preliminary results showed that the levels of fungal DNA ratios determined by qPCR approximately conformed to the performance of resistance in the field. For maize genotypes with low to intermediate levels of resistance, fungal DNA was detected as early as three days after inoculation. The detectable fungal DNA ratios increased over time until the end of the incubation period (when visible lesions formed), and thereafter decreased. This reduction in pathogen DNA was unexpected, and apparently corresponded to a loss of DNA integrity. DNA samples extracted from tissues showing lesions were usually brownish and of poor quality. The decrease in detectable fungal DNA ratio in highly diseased leaves is contradictory to our microscopic observation of abundant mycelial growth in NLB lesions. It is likely that in tissues with actively developing lesions, more DNA-degrading enzymes involving in cell death are present and can digest fungal DNA during the extraction. To better differentiate resistant and susceptible responses, it is critical to apply qPCR on infected tissues taken prior to the appearance of necrotic lesions on the most susceptible genotype in an experimental set.

Infected leaves from B73, Tx303, and two derived NILs were collected nine days after inoculation (according to the IP of the susceptible NIL), and measured for their fungal DNA content using $\mathrm{qPCR}$. As shown in Figure 6B, neither Tx303 nor $q N L B 1.06_{T \times 303}$ showed significant effect on reducing the growth of S. turcica in leaves. However, the fungal biomass ratio (\% of fungal DNA in the infected leaf) in the NIL with $q N L B 1.02_{T x 303}$ was $24 \%$ higher than in B73 $(P<0.0001)$, indicating that the in
(A)

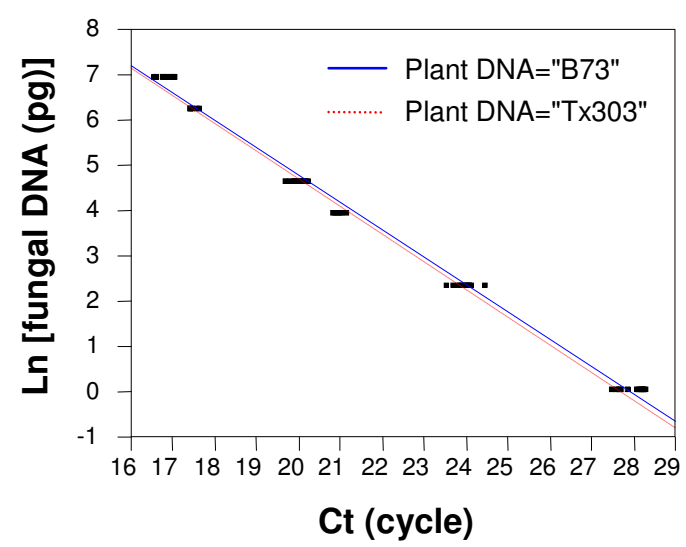

(B)

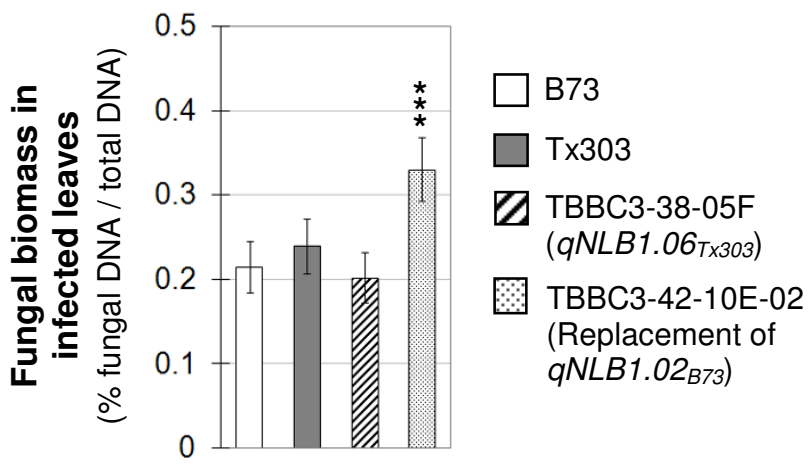

Figure 6 Quantifying QTL effect on mycelial growth of Setosphaeria turcica in planta using DNA-based real-time PCR. (A) Two standard curves were constructed by mixing a series of S. turcica DNA and 50 ng of maize DNA from non-inoculated B73 and Tx303 plants, respectively. With B73 DNA, Ln[fungal DNA] = 16.912- 0.606* Ct ( $\left.R^{2}=0.99\right)$; with Tx303 DNA, Ln[fungal DNA] = 16.906- $0.610^{*} \mathrm{Ct}\left(R^{2}=0.99\right)$. Ln: natural logarithm. Ct: threshold cycle, the number of PCR amplification cycle at which the exponential increase of the product was detected. (B) Measurement of S. turcica DNA ratio in infected leaves collected 9 dpi from maize genotypes B73 (open), Tx303 (gray), TBBC3-38-05F (hatched; the NIL carrying 9NLB1.06 $6_{\text {Tx303 }}$ ) and TBBC3-42$10 \mathrm{E}-02$ (dotted; the NIL carrying 9 NLB1.02 $2_{\text {T×303, }}$ which is essentially "replacement of $9 N L B 1.02_{B 73}$ "). Differences between least square means of different genotypes relative to B73 were determined by two-tailed Student's t test (significance level: ${ }^{*} 0.01<P<0.05 ;{ }^{* *} 0.001<P<0.01 ;{ }^{* * *} P<0.001$ ). The proportion data were arcsine transformed for statistical analysis, and the corresponding least squares means and $95 \%$ confidence intervals were backtransformed to original scale before plotting. The confidence intervals are bigger than significance levels due to asymmetry resulting from back transformation. 
planta development of $S$. turcica is more extensive on plants without $q N L B 1.02_{B 73}$.

\section{Preliminary characterization of potential QTL effects for multiple disease resistance using derived NILs}

Significant differences were observed between Tx303 and B73 for resistance to Stewart's wilt, anthracnose stalk rot (ASR), common smut and common rust. In the field, Tx303 was more resistant to Stewart's wilt and ASR, while B73 was more resistant to common smut and common rust. To investigate the resistance spectrum of $q N L B 1.02$ and $q N L B 1.06$, several NILs derived from B73 $\times$ TBBC3-38, B73 $\times$ TBBC3-39 and B3 $\times$ TBBC3-42 were evaluated for the four diseases (Additional files 4 and 5). The choice of NILs was constrained by the availability of seeds.

The evidence suggested that both $q N L B 1.06_{T \times 303}$ and $q N L B 1.02_{B 73}$ were effective for resistance to Stewart's wilt. As shown in Additional file 4, all the NILs carrying Tx303 introgression at bin 1.06 were significantly more resistant than B73. Based on comparing TBBC3-39-19E to $\mathrm{B} 73, q N L B 1.06_{T \times 303}$ was inferred to be effective for reducing lesions of Stewart's wilt on inoculated leaves by as much as $39 \%(P<0.0001)$. Likewise, in Additional file 5 , lines with Tx303 alleles at bin 1.01-1.02 were significantly more susceptible than B73. The effect of $q N L B 1.02_{B 73}$ on Stewart's wilt was estimated as reducing $19 \%$ of primary DLA based on a comparison of TBBC3$42-10 \mathrm{E}-02$ and B73 $(P<0.0001)$. Significant differences were also observed among the lines fixed (homozygous for Tx303 or B73 alleles) at bins 1.01-1.02, indicating that there are QTL other than $q N L B 1.02_{B 73}$ segregating in the NIL set. However, none of the rest of the introgressed regions were unambiguously associated with resistance.

qNLB1.06 ${ }_{T \times 303}$ was ineffective for resistance to ASR, smut and rust. No phenotypic difference in the development of rust and smut galls was detected among B73, TBBC3-38, TBBC3-39 and their derived NILs. Significant difference in discolored internode area was observed between TBBC3-38 and B73 $(\sim 32 \%, P=0.001)$, but the variation was not associated with qNLB1.06, as TBBC339 and the NIL carrying a single introgression at bin 1.06 (TBBC3-39-19E) showed the same resistance level as B73 (Additional file 4).

qNLB1.02 $2_{B 73}$ was ineffective for resistance to ASR and common smut, but potentially effective for resistance to common rust (Additional file 5). For ASR, some phenotypic difference was found among B73, TBBC3-42 and derived NILs, but none of the identified introgressed regions were associated with resistance. B73 was more resistant than Tx303 for common smut and common rust. For common smut, there were almost no ear galls or stalk galls observed on B73, TBBC3-42 and TBBC3-42$10 \mathrm{E}-04$, suggesting that introgressed segments in TBBC342 did not affect smut development. (More ear galls were scored on TBBC3-42-10E-04 in the 2008 trial, but the significance was marginal $(P=0.04)$, and the finding was not consistent with the 2007 result). For common rust, except for TBBC3-42-06F-2, lines with Tx303 alleles at bin 1.011.02 were consistently more susceptible than B73 for all three severity ratings (differed by 1.1-3.1 scales in severity, $P<0.025$ ) and AUDPC (differed by about 43-53 scaleday, $P<0.001)$. The result indicated the possibility of rust QTL at bins 1.01-1.02 and/or unidentified introgressed regions in TBBC3-42.

\section{Discussion}

\section{Identification of NLB QTL using TBBC3 introgression lines}

Two QTL for resistance to NLB, $q N L B 1.02_{B 73}$ and $q N L B 1.06_{T \times 303}$, were successfully identified, validated and characterized using a population of introgression lines and derived NILs. We applied a stepwise strategy that allowed phenotyping of informative NILs over a series of generations. A special field design, in which each row of CSSL/NIL arranged next to a row of B73, allowed accurate visual comparisons and a relatively uniform epidemic across the field. We detected and sequentially validated QTL using multiple disease components, in a full set of 82 lines, a subset of 15 selected lines, five selected $\mathrm{BC}_{4} \mathrm{~F}_{2}$ populations, and three sets of derived $\mathrm{BC}_{4} \mathrm{~F}_{3} / \mathrm{BC}_{4} \mathrm{~F}_{4}$ NILs. Our primary hypothesis that individual QTL affect distinct stage(s) of NLB development was ultimately confirmed using two selected NILs (compared to B73) in repeated greenhouse and field trials, with a panel of conventional and novel components. Our results revealed that $q N L B 1.06_{T \times 303}$ and $q N L B 1.02_{B 73}$ are mainly effective against the infection and colonization of $S$. turcica, respectively. We found that the QTL were both effective in both juvenile and adult plants, and that both chromosomal segments were associated with resistance to more than one disease.

The mechanism underlying resistance conferred by $q N L B 1.02_{B 73}$ and $q N L B 1.06_{T \times 303}$ is unlikely to be the same as the mechanism underlying qualitative resistance conferred by currently known major genes. Qualitative resistance to NLB is generally characterized by chloroticnecrotic lesions [65-69], chlorotic halo lesions [70], or extremely prolonged IP [71]. Considerable levels of resistance were seen for $q N L B 1.02_{B 73}$ and $q N L B 1.06_{T \times 303}$ in the two field sites, without observing distinct lesion type or much greater IP. Moreover, although co-localized NLB QTL at bins 1.02 and 1.06 have been reported in other populations $[72,73]$, no major genes have been mapped to these chromosomal regions. 


\section{Conventional and newly-developed components of resistance targeting different stages of NLB development}

QTL effects were analyzed using five conventional macroscopic disease parameters and four microscopic parameters. Each of the macroscopic components reflected different phase(s) of disease development. Incubation period quantifies the time to appearance of wilted lesions, which reflects the speed of xylem plugging due to extensive hyphal growth in the veins. Lesion expansion quantifies the rate of expansion of wilted and necrotic lesions, which reflects the speed of destructive hyphal growth in the leaves. Ratings for diseased leaf area, disease severity and area under the disease progress curve, on the other hand, involve visual quantification of overall disease progress on the entire plant. In our greenhouse and field trials, IP showed a better correlation than LE with DLA, disease severity and AUDPC. This indicates that IP is a more discriminating parameter than LE for measuring NLB severity. This confirms previous reports that IP is a convenient target trait in selection and breeding for resistance to NLB [11,73-78]. In a recurrent selection study for NLB resistance, selection for prolonged IP resulted in $\sim 7.5 \%$ more gain in reducing AUDPC per selection cycle compared to selection for lesion length [11].

QTL effects were also microscopically investigated at the pre-penetration, infection, and colonization phases. In most fungal pathosystems, pre-penetration resistance is associated with specialized physical and chemical features of plant surface which help reduce the incidence of landing, adhesion, germination, appressorium formation and penetration of pathogenic fungi [79]. Post-penetration resistance to microbial attack, on the other hand, is characterized by programmed necrosis, along with the induction of callose, lignin, phenolic compounds and other pathogenesis-related proteins around the primaryinfected cell. Four microscopic components, including the incidence of multiple appressoria, infection efficiency, accumulation of defense materials surrounding the infection site, and vascular invasion efficiency, were developed to complement the conventional macroscopic components. Modified trypan blue staining [25,42] and $\mathrm{KOH}-$ aniline blue fluorescence [43] methods were useful in determining the degree and timing of allelic contribution to quantitative resistance to NLB. Previous studies utilizing sectioning and electron microscopy have revealed that xylem plugging is a key stage for the formation of NLB lesions (Jennings and Ullstrup, 1957). Our microscopic examination using $\mathrm{KOH}$-aniline blue fluorescence technique confirmed the finding and allowed the observation of in planta colonization of S. turcica from a different angle. In all the maize genotypes examined, the mycelium appeared to grow preferentially towards vascular bundle, initially invading the vasculature, then extend- ing through the xylem vessel, and eventually, aggressively growing out to the neighboring mesophyll tissues. To our knowledge, the mechanisms of the post-infection directional growth of vascular fungal pathogens remain to be elucidated.

qPCR was employed for the first time for the measurement of in planta biomass of S. turcica. Although it has been widely applied in other pathosystems, qPCR can only be used for NLB quantification at early stages of pathogenesis. The accuracy and differentiating power are highly dependent on uniform inoculation and precise sampling at the early stages of pathogenesis, so the method is best suited to work conducted under controlled conditions. The biggest constraint of this technique comes from its not being able to measure the destructive mycelial growth in the blighted leaf areas, possibly due to the poor quality of DNA extracted from necrotic tissues. The cost of qPCR reagents and the required workload make it unfeasible for regular phenotypic screening. However, qPCR serves as a good tool for quantifying the degree of earlier mycelial colonization of S. turcica in maize leaves.

\section{Hypothesis \#1: individual QTL affect distinct stages of disease development}

Detailed macroscopic and microscopic evaluations revealed the distinct features of $q N L B 1.06_{T \times 303}$ and $q N L B 1.02_{B 73}$ in NLB development. $q N L B 1.06_{T \times 303}$ conditions resistance mainly against the penetration of $S$. turcica. The anti-penetration effect was observed at earlier but not later time points examined, indicating that $q N L B 1.06_{T \times 303}$ acts in a quantitative manner, which delays rather than prevents the occurrence of infection. In contrast, $q N L B 1.02_{B 73}$ appears to condition resistance not to infection, but rather by enhancing the induction of defense reactions surrounding infection sites, as well as by inhibiting hyphal growth into the vascular bundle, and the subsequent necrotrophic colonization in the leaves. It is difficult to infer whether the resistance is associated with defense reactions in mesophyll, parenchyma, bundle sheath and/or other cells.

Delaying the invasion and extension of S. turcica in the vascular system was shown to be critical for quantitative resistance to NLB. This is consistent with previous microscopic analyses [23,24] suggested that xylem invasion/plugging is an important pathogenetic phase in NLB progress. Polygenic resistance (in an inbred line C190A) has been associated with reduced hyphal spread into the xylem [22]. The significance of protecting the vasculature from hyphal invasion is supported by the evidence that the replacement of superior B73 allele with Tx303 allele at qNLB1.02 led to an $18-38 \%$ higher DLA (relative to B73). Incorporating a QTL effective in slowing down the inva- 
sion of the vasculature would thus considerably increase overall resistance.

We speculate that preventing the attachment of conidia and the subsequent infection plays a role in quantitative resistance to NLB in the donor line Tx303. Although this was not quantified, it was visually obvious that the number of spores per leaf segment of Tx303 was lower than the spore numbers on B73 or the NILs carrying $q N L B 1.06_{T \times 303}$ and $q N L B 1.02_{B 73}$. Lower infection efficiency of $S$. turcica was observed for Tx303 relative to B73. Interestingly, Tx303 showed a greater susceptibility than B73 for the parameters of vascular invasion efficiency, enhanced fluoresced area, and lesion expansion. Despite the lack of resistance in delaying vascular invasion and extension of S. turcica in Tx303, its higher effectiveness in reducing spore attachment and infection may contribute to the overall moderate resistance to NLB.

\section{Hypothesis \#2: the effectiveness of disease QTL is affected by plant maturity}

A number of maize diseases caused by hemibiotrophic fungi (eg. gray leaf spot, anthracnose leaf blight, anthracnose stalk rot) and necrotrophic fungi (eg. southern leaf blight) are known to progress more rapidly on the plants after anthesis $[18,49,80,81]$. In maize, a significant correlation has been detected between disease resistance and flowering time in a panel of 253 diverse lines (R. Wisser, J. Kolkman, P. Balint-Kurti, and R. Nelson, unpublished). The fact that flowering time may account for a considerable proportion of resistance variation leads to the hypotheses that the effects of disease QTL reflect indirect expression of flowering time QTL, and/or that the effects of disease QTL are modulated differently at varied plant developmental stages. In fact, several QTL identified in biparental populations are associated with both NLB resistance and flowering time (J. Poland, pers. comm.).

The association between NLB QTL and plant maturity was investigated in the subset of 15 selected TBBC3 lines. To separate resistance effects from maturity effects, plants in two different fields were inoculated at juvenile and adult stage (two weeks before tasselling) respectively, and evaluated for NLB resistance and flowering time. Generally consistent expression of resistance or susceptibility was observed at the two stages, implying that the effectiveness of these QTL was not altered substantially by plant maturity. While several of the introgression lines (10 out of the 15 TBBC 3 lines with differential resistance relative to $B 73$ ) showed significantly different flowering times relative to B73, the interactions between NLB QTL and flowering time QTL were relatively minor. For the $q N L B 1.02_{B 73}$ and $q N L B 1.06_{T \times 303}$, the analysis of advanced NIL sets further provided strong evidence of their independence from flowering time. This agrees with a previous report of flowering-time QTL in TBBC3 introgression lines [27]: bins 1.01-1.02 and 1.06 were not among the QTL affecting days to anthesis, days to silking, or anthesis-silking interval.

\section{Hypothesis \#3: the effectiveness of QTL is affected by environmental conditions}

A major limitation for QTL applications is the lack of consistency of QTL effects across environments. The inconsistent detection of QTL has been associated with experimental errors and differential gene expression affected by environmental factors [82]. In view of the widely reported inconsistency, the present study revealed that the introgressions/QTL with relatively large effects (carried in seven introgression lines: TBBC3-38, TBBC339, ТВВС3-42, ТВВС3-36, ТВВС3-21, ТВВС3-30, and TBBC3-77) were consistent in their performance across field sites and years. Reliable expression of these QTL, including $q N L B 1.02_{B 73}$ and $q N L B 1.06_{T \times 303}$, suggests their applicability to resistance breeding.

Disease resistance can be expressed differentially, not only in different field environments but also under field versus greenhouse conditions (eg. [80,83,84]). Differential efficacy of $q N L B 1.02_{B 73}$ and $q N L B 1.06_{T \times 303}$ in controlled greenhouse and field conditions was evaluated using macroscopic and microscopic phenotypes. It should be noted that due to the different inoculation and sampling treatments necessarily employed in different environments, the comparison between the effectiveness of disease QTL in the greenhouse and field should be addressed based on relative observation rather than the absolute values. Our overall results suggest that the resistance of $q N L B 1.02_{B 73}$ was stably expressed in the greenhouse and field, whereas $q N L B 1.06_{T \times 303}$ conferred a higher level of resistance in the field than in the greenhouse. Macroscopically, the effects of $q N L B 1.06_{T \times 303}$ on IP and primary DLA in greenhouse-grown plants were occasionally insignificant. Microscopically, the positive effect of $q N L B 1.06_{T \times 303}$ on triggering defense response surrounding the initially infected cell was only detected in the field. Greenhouse-grown plants carrying $q N L B 1.06_{T \times 303}$, however, displayed an opposite effect (smaller fluorescent area), which suggests that this type of resistance is highly affected by environmental factors. The observation that $q N L B 1.06_{T \times 303}$ increased the efficiency of $S$. turcica invasion into vasculature in the greenhouse but not field, may be an indirect effect of decreased accumulation of host defensive materials at the postinfection stage.

\section{Hypothesis \#4: QTL for resistance to NLB comprises genes or gene clusters involving in broad-spectrum resistance}

The phenomenon of multiple disease resistance (MDR) has been inferred based on the detection of QTL clusters 
affecting different diseases $[2,19,20,85]$, genetic correlations in populations [86], non-specific defense mechanisms (eg. SAR [87-89]), and genes (eg. npr1 [90] and $m l o$ [91]). Maize bins 1.02 and 1.06 have been previously associated with a number of disease QTL mapped in diverse maize populations [20]. Our evaluations of resistance to common rust, Stewart's wilt, anthracnose stalk rot, common smut, and common rust in sets of NILs suggest that both $q N L B 1.02_{B 73}$ and $q N L B 1.06_{T \times 303}$ encompass gene(s) contributing nonspecific defense effects. $q N L B 1.02_{B 73}$ was effective in decreasing NLB, common rust and Stewart's wilt, while $q N L B 1.06_{T \times 303}$ was effective in decreasing NLB and Stewart's wilt. Nevertheless, due to the low resolution of QTL localization in this study, it is unclear whether the nonspecific resistance of $q N L B 1.02_{B 73}$ and $q N L B 1.06_{T \times 303}$ is caused by pleiotropy or linkage, or whether their effects are mongenic or polygenic. Although the underlying mechanisms are unknown, the feature of broad-spectrum resistance makes the two identified disease QTL appealing in practical applications.

It is worth noting that the NILs carrying $q N L B 1.06_{T \times 303}$ showed remarkable resistance against Stewart's wilt, suggesting the involvement of major gene effect. In fact, a dominant major gene locus for Stewart's wilt, Sw1, has been mapped to bin 1.05-1.06 in inbred line Ki14 [92,93]. The co-localization of QTL for resistance to NLB and Stewart's wilt has also been observed in the NILs derived from the inbred line CML52 crossed to B73 (CML52 allele for resistance; C. Chung, unpublished).

\section{Conclusions}

Our research has led to successful identification of two reliably-expressed QTL that can potentially be utilized to protect maize from S. turcica in different environments. Map-based cloning will reveal more about the genes and mechanisms underlying the distinct features of $q N L B 1.02_{B 73}$ and $q N L B 1.06_{T \times 303}$ in the pre-penetration, penetration and post-penetration phases of pathogenesis. Large mapping populations have been generated from the NILs and fine-mapping of $q N L B 1.02_{B 73}$ and $q N L B 1.06_{T \times 303}$ is in progress.

\section{Additional material}

Additional file 1 NLB resistance of the full set of 82 TBBC 3 introgression lines. Relative area under the disease progress curve (Relative AUDPC) values shown are the differences of least squares means (from mixed models) between TBBC3 lines and B73 recurrent parent. AUDPC was calculated from three diseased leaf area (DLA) scores in the 2006 trial in NY (solid bars), or three disease severity scores in the 2006 trial in NC (open bars). In NY, primary DLA was also rated for diseased leaf area on inoculated leaves. The letters "R" and "S" below the graph indicate the lines significantly more resistant and more susceptible than $B 73$ at $P<0.05$, respectively, based on primary DLA and AUDPC. The 15 TBBC3 lines selected for subsequent phenotypic validation are indicated by rectangles highlighting the maize line designation
Additional file 2 Putative NLB QTL identified in the TBBC3 population. Putative QTL for northern leaf blight (NLB QTL) affecting incubation period $(I P)$, primary diseased leaf area (PrimDLA), diseased leaf area (DLA), disease severity (severity), and AUDPC (area under the disease progress curve calculated from DLA or disease severity) were identified using 82 TBBC3 introgression lines. QTL effects for each marker locus are the significant differences of least squares means of Tx303 homozygous genotypes at the locus relative to $B 73$ recurrent parent line $\left({ }^{*} 0.01<P<0.05\right.$, ${ }^{* *} 0.001<P<$ $\left.0.01{ }^{* * *} P<0.001\right)$. Putative QTL are reported as correlated groups because of the high dependencies among those introgressed segments in TBBC3 lines.

Additional file 3 Validation of NLB QTL in the $\mathrm{BC}_{4} \mathrm{~F}_{2}$ segregating populations. The $\mathrm{BC}_{4} \mathrm{~F}_{2}$ populations were genotyped and phenotyped for incubation period (IP), lesion expansion (LE), diseased leaf area (DLA), and area under the disease progress curve (AUDPC). The trait-marker association was tested by ANOVA at $P<0.05$. Introgressions/markers significantly associated with NLB resistance are listed. The relative allele effects are the differences on the least squares means (LSMean) between Tx303 homozygous genotypes and B73 homozygous genotypes at the locus.

Additional file 4 Genotypes and disease phenotypes for Tx303, B73 and the NIL sets derived from B73 $\times$ TBBC3-38 and B73 $\times$ TBBC3-39. Among the target introgressions at bins 1.03, 1.06, 3.02, 5.00, 5.02-5.03 and

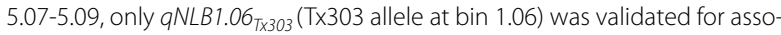
ciation with resistance to NLB. The open bars and solid bars represent the loci homozygous for B73 alleles and Tx303 alleles, respectively. The gray bars represent heterozygous loci or missing genotypic data. Only the chromosomes with introgressed regions in the two NIL sets are shown. The rest of the genome was assumed fixed for B73 alleles. Trait values are least squares means calculated from the mixed model. Pair-wise Student's t tests were performed to analyze the differences between each NIL and B73, and between every pair of NILs in each set. Trait values with different letters are significantly different from each other. Disease phenotypes that were significantly more resistant than B73 are highlighted in bold and shaded, while the phenotypes significant more susceptible than B73 are underscored. Lines that showed significantly different days to anthesis are in bold italic. qNLB1.06 $6_{T \times 303}$ was also effective for resistance to Stewart's wilt. (IP: incubation period; LE: lesion expansion; DLA: diseased leaf area; PrimDLA: primary DLA; AUDPC: area under the disease progress curve)

Additional file 5 Genotypes and disease phenotypes for Tx303, B73 and the NIL set derived from B73 $\times$ TBBC3-42. Among all target introgressions at bins 1.01-1.02, 4.06-4.07, 5.02-5.03, 7.01, 8.02 and 8.03-8.05, only qNLB1.02 ${ }_{B 73}$ (B73 allele at bin 1.02) was validated for association with resistance to NLB. The open bars and solid bars represent the loci homozygous for B73 alleles and Tx303 alleles, respectively. The gray bars represent heterozygous loci or missing genotypic data. Only the chromosomes with introgressed regions in the NIL sets are shown. The rest of the genome was assumed fixed for B73 alleles. Trait values are least squares means calculated from the mixed model. Pair-wise Student's t tests were performed to analyze the differences between each NIL and B73, and between every pair of NILs. Trait values with different letters are significantly different from each other. Disease phenotypes that were significantly more resistant than B73 are highlighted in bold and shaded, while the phenotypes significant more susceptible than B73 are underscored. Lines that showed significantly different days to anthesis are in bold italic. Preliminary evidence also suggested that $9 N L B 1.02_{B 73}$ was effective for resistance to Stewart's wilt, and QTL at bin 1.01-1.02 and/or 5.02-5.03 were associated with resistance to common rust.

\section{Authors' contributions}

CC and RN conceived of the study. CC designed and carried out all the experiments in New York, conducted statistical analysis, and drafted the manuscript. $J \mathrm{~L}$ and EW contributed to the design of microscopic analysis, participated in phenotypic data collection in the greenhouse and field trials in NY during 2007-2008. ZK participated in the inoculation and phenotypic data collection in the field trials in NY in 2008. GVE carried out the field trials in NC. PBK designed and carried out the field trials in NC, participated in coordination of the study, and contributed to its revision. RN participated in the coordination of the work and the experimental design, helped to draft the manuscript and contributed to its revision. All authors read and approved the final manuscript. 


\section{Acknowledgements}

We thank Jim Holland for providing the seeds of TBBC3 lines, Jesse Poland for help with rust inoculation, and Pioneer Hi-Bred International Inc. for the supply of sorghum grains used in production of inoculum. We appreciate help from Judith Kolkman, Oliver Ott and Kristen Kennedy with aspects in the research. The work was funded by The CGIAR Generation Challenge Program, The McKnight Foundation, USDA-ARS, and Ministry of Education, Taiwan.

\section{Author Details}

'Dept. of Plant Pathology and Plant-Microbe Biology, Cornell University, Ithaca, NY 14853, USA, 2Dept. of Plant Breeding and Genetics, Cornell University, Ithaca, NY 14853, USA, ${ }^{3}$ Dept. of Biology, West Virginia State University, Institute, WV 25112, USA, ${ }^{4}$ Dept. of Crop Science, North Carolina State University, Raleigh, NC 27695, USA and 5USDA-ARS, Plant Science Research Unit; Dept. of Plant Pathology, North Carolina State University, Raleigh, NC 27695, USA

Received: 16 January 2010 Accepted: 8 June 2010

Published: 8 June 2010

\section{References}

1. Jones JDG, Dangl JL: The plant immune system. Nature 2006, 444(7117):323-329.

2. Wisser RJ, Sun Q, Hulbert SH, Kresovich S, Nelson RJ: Identification and characterization of regions of the rice genome associated with broadspectrum, quantitative disease resistance. Genetics 2005,

169(4):2277-2293.

3. Manosalva PM, Davidson RM, Liu B, Zhu X, Hulbert SH, Leung H, Leach JE: A germin-Like protein gene family functions as a complex quantitative trait locus conferring broad-spectrum disease resistance in rice. Plant Physiol 2009, 149(1):286-296.

4. Krattinger SG, Lagudah ES, Spielmeyer W, Singh RP, Huerta-Espino J, McFadden $H$, Bossolini E, Selter LL, Keller B: A putative ABC transporter confers durable resistance to multiple fungal pathogens in wheat. Science 2009, 323(5919):1360-1363.

5. Fukuoka S, Saka N, Koga H, Ono K, Shimizu T, Ebana K, Hayashi N, Takahashi A, Hirochika H, Okuno K, Yano M: Loss of function of a prolinecontaining protein confers durable disease resistance in rice. Science 2009, 325:998-1001.

6. Poland JA, Balint-Kurti PJ, Wisser RJ, Pratt RC, Nelson RJ: Shades of gray: the world of quantitative disease resistance. Trends Plant Sci 2009, 14(1):21-29.

7. Fu D, Uauy C, Distelfeld A, Blechl A, Epstein L, Chen X, Sela H, Fahima T, Dubcovsky J: A Kinase-START gene confers temperature-dependent resistance to wheat stripe rust. Science 2009, 323(5919):1357-1360.

8. Parlevliet J: Durability of resistance against fungal, bacterial and viral pathogens; present situation. Euphytica 2002, 124:147-156.

9. Young ND: QTL mapping and quantitative disease resistance in plants. Annu Rev Phytopathol 1996:479-501.

10. Broglie KE, Butler KH, Butruille MG, da Silva Conceicao A, Frey TJ, Hawk JA, Jaqueth JS, Jones ES, Multani DS, Wolters PJCC, E.I. du Pont de Nemours and Company, Pioneer Hi-Bred International, Inc., University of Delaware: Polynucleotides and methods for making plants resistant to fungal pathogens. United States Patent 20060223102. United States Patent and Trademark Office 2006.

11. Carson ML: Response of a maize synthetic to selection for components of partial resistance to Exserohilum turcicum. Plant Dis 2006, 90(7):910-914.

12. Vidhyasekaran P: Fungal Pathogenesis in Plants and Crops: Molecular Biology and Host Defense Mechanisms. 2nd edition. Boca Raton, FL: CRC Press; 2007

13. Moldenhauer J, Pretorius ZA, Moerschbacher BM, Prins R, Van Der Westhuizen AJ: Histopathology and PR-protein markers provide insight into adult plant resistance to stripe rust of wheat. Mol Plant Pathol 2008, 9(2):137-145.

14. Ullstrup AJ, Miles SR: The effects of some leaf blights of corn on grain yield. Phytopathology 1957, 47(6):331-336.

15. Raymundo $A D$, Hooker $A L$ : Measuring the relationship between northern corn leaf blight and yield losses. Plant Dis 1981, 65(4):325-327.
16. Perkins JM, Pedersen WL: Disease development and yield losses associated with northern leaf blight on corn. Plant Dis 1987 71(10):940-943.

17. Pingali PL, Pandey S: Meeting world maize needs: technological opportunities and priorities for the public sector. In CIMMYT 1999/2000 World Maize Facts and Trends Edited by: Pingali PL, Mexico DF. CIMMYT; 2001

18. Carson ML: Helminthosporium leaf spots and blights. In Compendium of Corn Diseases 3rd edition. Edited by: White DG. St. Paul, Minnesota: The American Phytopathology Society; 1999:15-24.

19. Welz HG, Geiger HH: Genes for resistance to northern corn leaf blight in diverse maize populations. Plant Breed 2000, 119(1):1-14.

20. Wisser RJ, Balint-Kurti PJ, Nelson RJ: The genetic architecture of disease resistance in maize: a synthesis of published studies. Phytopathology 2006, 96(2):120-129.

21. Pratt RC, Gordon SG: Breeding for resistance to maize foliar pathogens. Plant Breed Rev 2006, 27:119-173.

22. Hilu HM, Hooker AL: Host-pathogen relationship of Helminthosporium turcicum in resistant and susceptible corn seedlings. Phytopathology 1964, 54(5):570-575

23. Hilu HM, Hooker AL: Localized infection by Helminthosporium turcicum on corn leaves. Phytopathology 1965, 55(2):189-192.

24. Jennings PR, Ullstrup AJ: A histological study of three Helminthosporium leaf blights of corn. Phytopathology 1957, 47(12):707-714

25. Knox-Davies PS: Penetration of maize leaves by Helminthosporium turcicum. Phytopathology 1974, 64(11):1468-1470.

26. Stuber CW, Polacco M, Senior ML: Synergy of empirical breeding, marker-assisted selection, and genomics to increase crop yield potential. Crop Sci 1999, 39(6):1571-1583.

27. Szalma SJ, Hostert BM, LeDeaux JR, Stuber CW, Holland JB: QTL mapping with near-isogenic lines in maize. Theor App/ Genet 2007, 114(7):1211-1228.

28. Li Z-K, Fu B-Y, Gao Y-M, Xu J-L, Ali J, Lafitte HR, Jiang Y-Z, Rey JD, Vijayakumar CHM, Maghirang R, Zheng T-Q, Zhu L-H: Genome-wide introgression lines and their use in genetic and molecular dissection of complex phenotypes in rice (Oryza sativa L.). Plant Mol Biol 2005, 59(1):33-52.

29. Toojinda T, Baird E, Booth A, Broers L, Hayes P, Powell W, Thomas W, Vivar $H$, Young $G$ : Introgression of quantitative trait loci (QTLs) determining stripe rust resistance in barley: an example of marker-assisted line development. Theor App/ Genet 1998, 96(1):123-131.

30. Brown AHD, Munday J, Oram RN: Use of isozyme-marked segments from wild barley (Hordeum spontaneum) in barley breeding. Plant Breed 1988, 100(4):280-288.

31. Eshed Y, Zamir D: An introgression line population of Lycopersicon pennellii in the cultivated tomato enables the identification and fine mapping of yield-associated QTL. Genetics 1995, 141(3):1147-1162.

32. Keurentjes JJB, Bentsink L, Alonso-Blanco C, Hanhart CJ, Vries HB-D, Effgen $S$, Vreugdenhil D, Koornneef M: Development of a near-isogenic line population of Arabidopsis thaliana and comparison of mapping power with a recombinant inbred line population. Genetics 2007, 175(2):891-905

33. Kaeppler SM: Quantitative trait locus mapping using sets of nearisogenic lines: relative power comparisons and technical considerations. Theor App/ Genet 1997, 95(3):384-392.

34. Remington DL, Purugganan MD: Candidate genes, quantitative trait loci, and functional trait evolution in plants. Int J Plant Sci 2003, 164(3 Supplement):S7-S20.

35. Whalen MC: Host defence in a developmental context. Mol Plant Pathol 2005, 6(3):347-360.

36. Kim KD, Hwang BK, Koh YJ: Evaluation of rice cultivars under greenhouse conditions for adult-plant resistance to Pyricularia oryzae. J Phytopathol 1987, 120(4):310-316.

37. Century KS, Lagman RA, Adkisson M, Morlan J, Tobias R, Schwartz K, Smith A, Love J, Ronald PC, Whalen MC: Developmental control of Xa21mediated disease resistance in rice. Plant J 1999, 20(2):231-236.

38. Kus JV, Zaton K, Sarkar R, Cameron RK: Age-related resistance in Arabidopsis is a developmentally regulated defense response to Pseudomonas syringae. Plant Cell 2002, 14(2):479-490. 
39. Collins A, Milbourne D, Ramsay L, Meyer R, Chatot-Balandras C, Oberhagemann P, De Jong W, Gebhardt C, Bonnel E, Waugh R: QTL for field resistance to late blight in potato are strongly correlated with maturity and vigour. Mol Breed 1999, 5(5):387-398.

40. Kumar JR, Kumar BT: Quantitative trait loci (QTL) mapping for crop improvement. Res J Biotech 2009, 4(2):67-79.

41. Leonard KJ, Levy Y, Smith DR: Proposed nomenclature for pathogenic races of Exserohilum turcicum on corn. Plant Dis 1989, 73(9):776-777.

42. Vélez H: Alternaria alternata mannitol metabolism in plant-pathogen interactions. In PhD thesis North Carolina State University, Department of Plant Pathology; 2005.

43. Hood ME, Shew HD: Applications of $\mathrm{KOH}$-aniline blue fluorescence in the study of plant-fungal interactions. Phytopathology 1996, 86(7):704-708

44. Qi M, Yang Y: Quantification of Magnaporthe grisea during infection of rice plants using real-time polymerase chain reaction and northern blot/phosphoimaging analyses. Phytopathology 2002, 92(8):870

45. Gaurilcikiene I, Deveikyte I, Petraitiene E: Epidemic progress of Cercospora beticola Sacc. in Beta vulgaris L. under different conditions and cultivar resistance. Biologija 2006, 4:54-59.

46. Pataky JK: Influence of host resistance and growth stage at the time of inoculation on Stewart's wilt and Goss's wilt development and sweet corn hybrid yield. Plant Dis 1989, 73(4):339-345.

47. Blanco MH, Johnson MG, Colbert TR, Zuber MS: An inoculation technique for Stewart's wilt disease of corn. Plant Dis Rep 1977, 61(5):413-416.

48. Chang C-M, Hooker AL, Lim SM: An inoculation technique for determining Stewart's bacterial leaf blight reaction in corn. Plant Dis Rep 1977, 61(12):1077-1079.

49. Keller NP, Bergstrom GC: Development predisposition of maize to anthracnose stalk rot. Plant Dis 1988, 72(11):977-980.

50. Muimba-Kankolongo A, Bergstrom GC: Transitory wound predisposition of maize to Anthracnose stalk rot. Can J Plant Pathol 1990, 12(1):1-10.

51. du Toit LJ, Pataky JK: Variation associated with silk channel inoculation for common smut of sweet corn. Plant Dis 1999, 83(8):727-732

52. Webb CA, Richter TE, Collins NC, Nicolas M, Trick HN, Pryor T, Hulbert SH: Genetic and molecular characterization of the maize $r p 3$ rust resistance locus. Genetics 2002, 162(1):381-394.

53. Pataky JK, Campana MA: Reduction in common rust severity conferred by the $R p 1 D$ gene in sweet corn hybrids infected by mixtures of $R p 1 D$ virulent and avirulent Puccinia sorghi. Plant Dis 2007, 91(11):1484-1488.

54. Doyle JJ, Doyle JL: A rapid DNA isolation procedure for small quantities of fresh leaf tissue. Phytochem Bull 1987, 19:11-15.

55. Qiu F, Wang H, Chen J, Zhuang J, Hei L, Cheng S, Wu J: A rapid DNA miniprep method for large-scale rice mutant screening. Rice Sci 2006, 13(4):299-302.

56. Schuelke M: An economic method for the fluorescent labeling of PCR fragments. Nat Biotechno/ 2000, 18(2):233-234

57. Wisser RJ, Murray SC, Kolkman JM, Ceballos H, Nelson RJ: Selection mapping of loci for quantitative disease resistance in a diverse maize population. Genetics 2008, 180(1):583-599.

58. Patterson HD, Williams ER: A new class of resolvable incomplete block designs. Biometrika 1976, 63(1):83-92.

59. van Dam J, Levin I, Struik PC, Levy D: Identification of epistatic interaction affecting glycoalkaloid content in tubers of tetraploid potato (Solanum tuberosum L.). Euphytica 2003, 134(3):353-360.

60. Gonzalez J, Reyes F, Salas C, Santiago M, Codriansky Y, Coliheuque N, Silva $\mathrm{H}$ : Arabidopsis thaliana: A model host plant to study plant-pathogen interaction using Chilean field isolates of Botrytis cinerea. Biol Res 2006, 39(2):221-228.

61. Johnston PR, Sutherland PW, Joshee S: Visualising endophytic fungi within leaves by detection of (1->3)-beta-D-glucans in fungal cell walls. Mycologist 2006, 20(4):159-162.

62. Soylu S: Accumulation of cell-wall bound phenolic compounds and phytoalexin in Arabidopsis thaliana leaves following inoculation with pathovars of Pseudomonas syringae. Plant Sci 2006, 170(5):942-952.

63. Ficke A, Gadoury DM, Seem RC: Ontogenic resistance and plant disease management: A case study of grape powdery mildew. Phytopathology 2002, 92(6):671-675

64. Bennett M, Gallagher M, Fagg J, Bestwick C, Paul T, Beale M, Mansfield J: The hypersensitive reaction, membrane damage and accumulation of autofluorescent phenolics in lettuce cells challenged by Bremia lactucae. Plant J 1996, 9(6):851-865

65. Hooker AL: Inheritance of chlorotic-lesion resistance to Helminthosporium turcicum in seedling corn. Phytopathology 1963, 53(6):660-662.

66. Hooker AL: A second major gene locus in corn for chlorotic lesion resistance to Helminthosporium turcicum. Crop Sci 1977, 17(1):132-135.

67. Hooker AL: Resistance to Helminthosporium turcicum from Tripsacum floridanum incorporated into corn. Maize Genet Coop News/ 1981, 55:87-88.

68. Ogliari JB, Guimaraes MA, Geraldi IO, Aranha Camargo LE: New resistance genes in the Zea mays - Exserohilum turcicum pathosystem. Genet Mol Biol 2005, 28(3):435-439.

69. Ogliari JB, Guirnaraes MA, Aranha Carnargo LE: Chromosomal locations of the maize (Zea mays L.) HtP and $r t$ genes that confer resistance to Exserohilum turcicum. Genet Mol Biol 2007, 30(3):630-634.

70. Carson ML: A new gene in maize conferring the "chlorotic halo" reaction to infection by Exserohilum turcicum. Plant Dis 1995, 79(7):717-720.

71. Gevers HO: A new major gene for resistance to Helminthosporium turcicum leaf blight of maize. Plant Dis Rep 1975, 59(4):296-299.

72. Freymark PJ, Lee M, Woodman WL, Martinson CA: Quantitative and qualitative trait loci affecting host-plant response to Exserohilum turcicum in maize (Zea mays L.). Theor App/ Genet 1993, 87(5):537-544

73. Welz HG, Xia XC, Bassetti P, Melchinger AE, Luebberstedt T: QTLs for resistance to Setosphaeria turcica in an early maturing Dent $\times$ Flint maize population. Theor App/ Genet 1999, 99(3-4):649-655.

74. Carson ML: Inheritance of latent period length in maize infected with Exserohilum turcicum. Plant Dis 1995, 79(6):581-585.

75. Smith DR, Kinsey JG: Latent period - a possible selection tool for Exserohilum turcicum resistance in corn (Zea mays L.). Maydica 1993, 38:205-208

76. Welz HG, Schechert AW, Geiger HH: Dynamic gene action at QTLs for resistance to Setosphaeria turcica in maize. Theor App/ Genet 1999, 98(67):1036-1045.

77. Schechert AW, Welz HG, Geiger HH: QTL for resistance to Setosphaeria turcica in tropical African maize. Crop Sci 1999, 39(2):514-523.

78. Brewster VA, Carson ML, Wicks ZW III: Mapping components of partial resistance to northern leaf blight of maize using reciprocal translocation. Phytopathology 1992, 82(2):225-229.

79. Tucker SL, Talbot NJ: Surface attachment and pre-penetration stage development by plant pathogenic fungi. Annu Rev Phytopathol 2001:385-417.

80. Bubeck DM, Goodman MM, Beavis WD, Grant D: Quantitative trait loci controlling resistance to gray leaf spot in maize. Crop Sci 1993, 33(4):838-847

81. Leonard KJ, Thompson DL: Effects of temperature and host maturity on lesion development of Colletotrichum graminicola on corn. Phytopathology 1976, 66(5):635-639.

82. Sofi P, Rather AG: QTL analysis in rice improvement: concept, methodology and application. Biotechnology 2007, 6(1):1-13.

83. Trognitz BR, Orrillo M, Portal L, Roman C, Ramon P, Perez S, Chacon G: Evaluation and analysis of reduction of late blight disease in a diploid potato progeny. Plant Pathol 2001, 50(3):281-291.

84. Dinh SQ, Joyce DC, Irving DE, Wearing AH: Effects of combined methyl jasmonate and ethylene-inhibitor treatments against Botrytis cinerea infecting Geraldton waxflower. Acta Hort (ISHS) 2007, 755:527-533.

85. Balint-Kurti PJ, Wisser R, Zwonitzer JC: Use of an advanced intercross line population for precise mapping of quantitative trait loci for gray leaf spot resistance in maize. Crop Sci 2008, 48(5):1696-1704

86. Mitchell-Olds T, James RV, Palmer MJ, Williams PH: Genetics of Brassica rapa (syn. campestris). 2. Multiple disease resistance to three fungal pathogens. Peronospora parasitica, Albugo candida and Leptosphaeria maculans. Heredity 1995, 75(4):362-369.

87. Asai T, Tena G, Plotnikova J, Willmann MR, Chiu W-L, Gomez-Gomez L, Boller T, Ausubel FM, Sheen J: MAP kinase signalling cascade in Arabidopsis innate immunity. Nature 2002, 415(6875):977-983.

88. Durrant WE, Dong X: Systemic acquired resistance. Annu Rev Phytopathol 2004, 42:185-209.

89. Yang Y, Shah J, Klessig DF: Signal perception and transduction in plant defense responses. Gene Dev 1997, 11(13):1621-1639. 
90. Mou Z, Fan W, Dong X: Inducers of plant systemic acquired resistance regulate NPR1 function through redox changes. Cell 2003,

113(7):935-944

91. Consonni C, Humphry ME, Hartmann HA, Livaja M, Durner J, Westphal L, Vogel J, Lipka V, Kemmerling B, Schulze-Lefert P, Somerville SC, Panstruga $\mathrm{R}$ : Conserved requirement for a plant host cell protein in powdery mildew pathogenesis. Nat Genet 2006, 38(6):716-720.

92. Pataky JK, Bohn MO, Lutz JD, Richter PM: Selection for quantitative trait loci associated with resistance to Stewart's wilt in sweet corn. Phytopathology 2008, 98(4):469-474.

93. Ming R, Brewbaker JL, Moon HG, Musket TA, Holley RN, Pataky JK, MCMullen MD: Identification of RFLP makers linked to a major gene, sw1, conferring resistance to Stewart's wilt in maize. Maydica 1999, 44:319-323

94. Freymark PJ, Lee M, Martinson CA, Woodman WL: Molecular-markerfacilitated investigation of host-plant response to Exserohilum turcicum in maize (Zea mays L.): components of resistance. Theor App/ Genet 1994, 88(3-1):305-313.

95. Carson ML, Van Dyke CG: Effect of light and temperature on expression of partial resistance of maize to Exserohilum turcicum. Plant Dis 1994, 78(5):519-522.

96. Sigulas KM, Hill RRJ, Ayers JE: Genetic analysis of Exserohilum turcicum lesion expansion on corn. Phytopathology 1988, 78(2):149-153.

97. Brown AF, Juvik JA, Pataky JK: Quantitative trait loci in sweet corn associated with partial resistance to Stewart's wilt, northern corn leaf blight, and common rust. Phytopathology 2001, 91(3):293-300.

98. Balint-Kurti PJ, Zwonitzer JC, Wisser RJ, Carson ML, Oropeza-Rosas MA, Holland JB, Szalma SJ: Precise mapping of quantitative trait loci for resistance to southern leaf blight, caused by Cochliobolus heterostrophus race $\mathrm{O}$, and flowering time using advanced intercross maize lines. Genetics 2007, 176(1):645-657.

\section{doi: $10.1186 / 1471-2229-10-103$}

Cite this article as: Chung et al., Resistance loci affecting distinct stages of fungal pathogenesis: use of introgression lines for QTL mapping and characterization in the maize - Setosphaeria turcica pathosystem BMC Plant Biology 2010, 10:103

Submit your next manuscript to BioMed Central and take full advantage of:

- Convenient online submission

- Thorough peer review

- No space constraints or color figure charges

- Immediate publication on acceptance

- Inclusion in PubMed, CAS, Scopus and Google Scholar

- Research which is freely available for redistribution

Submit your manuscript at www.biomedcentral.com/submit 